\title{
Review
}

Yuxin Yan, Fathima Zahra Nashath, Sharon Chen, Sivakumar Manickam, Siew Shee Lim, Haitao Zhao, Edward Lester, Tao Wu, and Cheng Heng Pang*

\section{Synthesis of graphene: Potential carbon precursors and approaches}

https://doi.org/10.1515/ntrev-2020-0100

received September 28, 2020; accepted November 28, 2020

Abstract: Graphene is an advanced carbon functional material with inherent unique properties that make it suitable for a wide range of applications. It can be synthesized through either the top-down approach involving delamination of graphitic materials or the bottom-up approach involving graphene assembly from smaller building units. Common top-down approaches are exfoliation and reduction while bottom-up approaches include chemical vapour deposition, epitaxial growth, and pyrolysis. A range of materials have been successfully used as precursors in various synthesis methods to derive graphene. This review analyses and discusses the suitability

\footnotetext{
* Corresponding author: Cheng Heng Pang, Department of Chemical and Environmental Engineering, University of Nottingham Ningbo China, Ningbo 315100, China; Municipal Key Laboratory of Clean Energy Conversion Technologies, University of Nottingham Ningbo China, Ningbo 315100, China,

e-mail: ChengHeng.Pang@nottingham.edu.cn

Yuxin Yan: Key Laboratory for Carbonaceous Wastes Processing and Process Intensification Research of Zhejiang Province, University of Nottingham Ningbo China, Ningbo, 315100, China

Fathima Zahra Nashath, Siew Shee Lim: Department of Chemical and Environmental Engineering, University of Nottingham Malaysia, Broga Road, Semenyih, 43500, Selangor, Malaysia

Sharon Chen: Department of Chemical and Environmental Engineering, University of Nottingham Ningbo China, Ningbo 315100, China

Sivakumar Manickam: Petroleum and Chemical Engineering, Faculty of Engineering, Universiti Teknologi Brunei, Bandar Seri Begawan, BE1410, Brunei Darussalam

Haitao Zhao: Department of Mechanical Engineering, Massachusetts Institute of Technology, Cambridge, MA 02139, United States of America

Edward Lester: Department of Chemical and Environmental Engineering, The University of Nottingham, Nottingham NG7 2RD, United Kingdom

Tao Wu: Key Laboratory for Carbonaceous Wastes Processing and Process Intensification Research of Zhejiang Province, University of Nottingham Ningbo China, Ningbo, 315100, China; New Materials Institute, The University of Nottingham Ningbo China, Ningbo 315100, China
}

of conventional, plant- and animal-derived, chemical, and fossil precursors for graphene synthesis. Together with its associated technical feasibility and economic and environmental impacts, the quality of resultant graphene is critically assessed and discussed. After evaluating the parameters mentioned above, the most appropriate synthesis method for each precursor is identified. While graphite is currently the most common precursor for graphene synthesis, several other precursors have the potential to synthesize graphene of comparable, if not better, quality and yield. Thus, this review provides an overview and insights into identifying the potential of various carbon precursors for large-scale and commercial production of fit-for-purpose graphene for specific applications.

Keywords: graphite, top-down, bottom-up, biomass, polymer, coal, glucose, ethanol, cheese, methane

\section{Introduction}

Since its first recorded observation in 1859, graphene has been lauded for its unique morphological, electronic, and mechanical properties resulting in a wide range of applications. Graphene is a single layer of $\mathrm{sp}^{2}$ hybridized carbon atoms in a hexagonal arrangement with $\pi$-electron clouds [1]. First isolated in the form of graphene oxide (GO) by chemist Benjamin C. Brodie, the material was produced by the addition of potassium chlorate to a slurry of graphite in fuming nitric acid [2]. However, methods to effectively derive graphene in monolayers did not prove successful until the Scotch tape method [3], which utilizes micromechanical exfoliation and was developed in 2004 by Geim and Novoselov. In addition to winning the Nobel Prize in Physics in 2010 [4], their relatively simple, yet effective, method sparked an increased interest in graphene research to further explore its properties and applications [5].

Industrial-scale graphene production still faces many issues mainly due to technological immaturity and, thus, 
costly synthesis [6]. The scale-up from laboratory to commercial plant is a sensitive process as the quality and properties of graphene largely rely on the processing facilities and synthesis parameters, thus leading to uniformity and reproducibility issues. Furthermore, the raw graphite used for exfoliation may be sourced differently with differing quality, impurities, and morphologies, which also affect the uniformity of the final graphene product [7]. At the same time, these issues result in products drifting away from pristine graphene and causing structural defects that significantly affect its electronic and mechanical properties [8]. These defects may prove useful in applications such as biomedical devices that function through the attachment of molecules to the defects or to enhance the chemical reactivity of graphene through the defects associated with dangling bonds [9]. A study on graphene sourced from several manufacturers not only demonstrates large variations in its properties but also concludes multiple products were not suitable for most graphene applications [10]. Due to the large variations in the quality of graphene across suppliers, there have been calls [7] for a universally accepted, standardized grading system for graphene products similar to that of the carbon fibre. Thickness, uniformity, defects, and contamination levels need to be controlled under this system and can be assessed through microscopy and UV irradiation [11]. With the world's graphene market expected to exceed $€ 150$ million by 2022 [7], these industrial challenges must be overcome to realize the potential of graphene.

Graphene's potential in various applications arises owing to its unique properties. Graphene is 40 times stronger than diamond with high tensile strength of $130 \mathrm{GPa}[12,13]$. This property is utilized in the production of graphene-reinforced bulletproof vests, as traditional Kevlar vests have low compression strength and its considerable weight hinders the user's movements. Graphene is incorporated between Kevlar layers to increase the ballistic performance while not significantly increasing the weight of the vest [14]. In construction studies, incorporating just $0.04 \mathrm{wt} \%$ [15] of GO in cement-based materials greatly improves strength, which is useful for rapid repair engineering applications [16] and imparts chloride penetration resistance, which increases the durability of concrete [17]. In a similar vein, graphene can be incorporated into rubber asphalt, resulting in better hightemperature rutting resistance and lower temperature sensitivity that provide enhanced durability for roads experiencing heavy traffic [18]. GO was found to impart greater compressive strength to sulphoaluminate cement (SAC), particular as curing time is increased as displayed

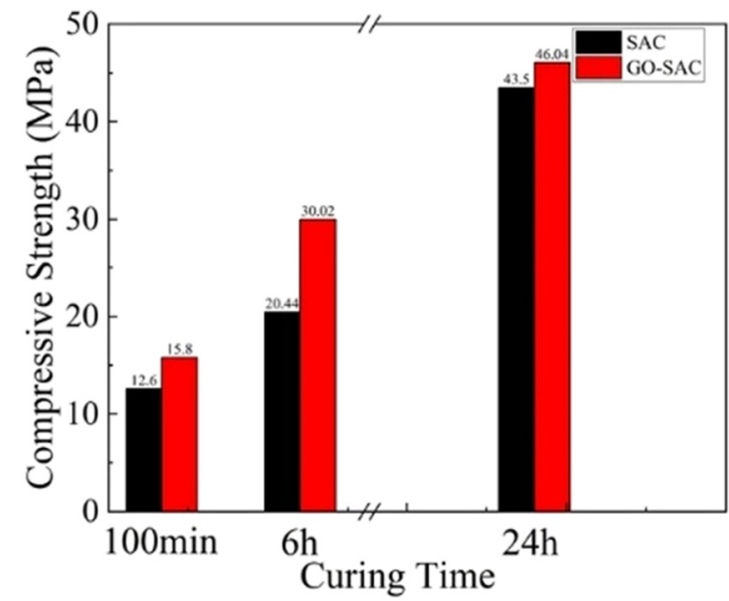

Figure 1: Compressive strength of GO-SAC and SAC at increasing curing times [15].

in Figure 1 [15]. Graphene is also used as a toughening modifier for polymers to impart strength to polymer composites [19]. Graphene-reinforced polymers have thermal conductivities superior to those incorporating carbon nanotubes (CNTs) [20] and higher electrical conductivities than conventional electrodes such as gold and platinum [21]. As detailed in Figure 2 [20], not only do graphene polymers display higher thermal conductivities than CNT polymers, its thermal conductivity can be further improved by increasing the nanofiller content. Incorporating graphene into a ceramic matrix is advantageous as it helps address conventional ceramic's brittleness and low fracture toughness [22]. This strength mainly occurs due to crack bridging as illustrated in Figure 3, wherein the graphene deflects the crack at an angle, thus consuming the fracture energy and reducing the crack-propagation rate. More recently, it has been suggested that zirconia-graphene ceramics portray

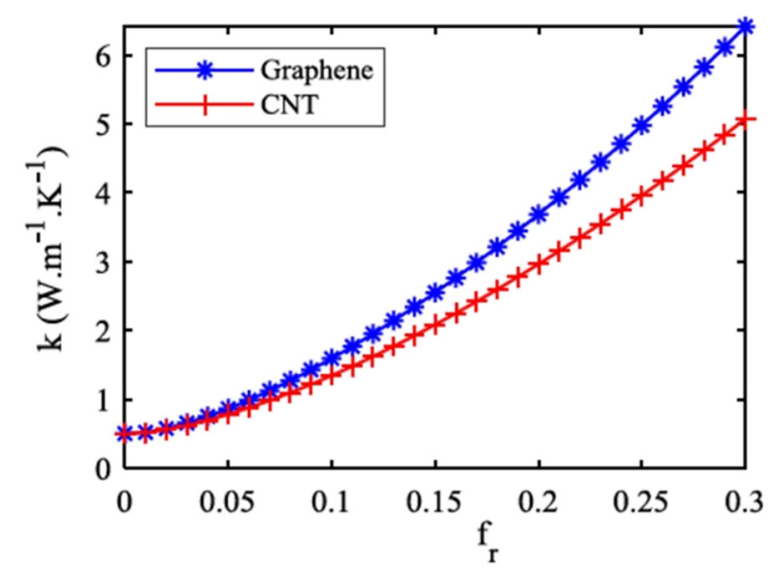

Figure 2: Thermal conductivities of graphene and CNT polymers versus volume fraction of nanofillers [20]. 


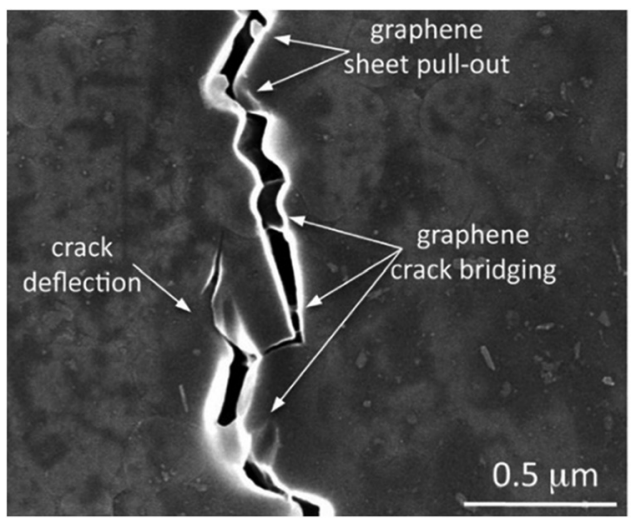

Figure 3: Toughening mechanism in graphene ceramic matrix composite [22].

enhanced hardness and crack resistivities, yet its application as functional high-temperature materials is still debatable [23]. Graphene is also known to strengthen metallic composites, with $0.1 \mathrm{wt} \%$ graphene increasing the ultimate tensile strength of copper by 1.8 times [24]. Graphene's strength, hardness, and self-lubricating properties allow it to be used as secondary reinforcement in the synthesis of light weight self-lubricating composites for aerospace and automobile applications [25]. In addition, graphene has a large specific surface area theoretically calculated to be $2,418 \mathrm{~m}^{2} \mathrm{~g}^{-1}$ [26]. This allows graphene to be more efficient at storing electrostatic charges, making it suitable for supercapacitor applications [27]. Apart from energy storage, the high specific surface area is also advantageous in catalysis [28], adsorption [29], and water purification [30]. While most catalysts are metallic compounds, they are frequently expensive, toxic, polluting, and run the risk of severe aggregation due to high surface energy, thus reducing their catalytic activity [31]. Graphene aerogels were found to be effective in the adsorption of oils, dyes and organic solvents from an aqueous medium [32]. Moreover, graphene shows high transparency in visible light, allowing up to $97.7 \%$ of irradiated light to pass through it, enabling its use in photovoltaic devices such as solar cells [33-35] and the fabrication of transparent conducting electrodes [36]. Furthermore, graphene is hydrophobic, allowing it to be used in nanocomposite coatings for antifouling applications [37]. Graphene has a high electrical conductivity due to the free movement of electrons in the $\pi$-electron clouds and a high thermal conductivity due to the strong bonding of its carbon atoms $[38,39]$. Furthermore, a molecular dynamics simulation [40] revealed monolayer graphene displays auxeticity, particularly at increasing temperatures, which enhances graphene's macroscopic properties such as indentation resistance.

$\mathrm{GO}$ and its reduced form ( $\mathrm{rGO}$ ) have been studied intensively as alternatives to graphene due to the relative ease by which their productions can be scaled up. In some applications, GO is preferred due to graphene's tendency to aggregate, resulting in low dispersions in liquid media. GO consists of different oxygen functional groups, including epoxy, carboxyl, carbonyls, and hydroxyl groups [41]. These oxygen functional groups offer superhydrophilic properties to GO for water permeability [42]. GO also exhibits good biocompatibility and high affinity for specific biomolecules which are essential for biomedical applications [43]. While GO is an insulating material, $\mathrm{rGO}$ is conductive but exhibits strongly reduced conductivity compared to mechanically exfoliated graphene. This is due to electronic transport in rGO occurring via electron hopping over varying distances between the non-oxidized part of the GO instead of direct electron flow through the $\pi$-electron clouds in graphene [44]. Recently, rGO has been applied to the reduction of electromagnetic wave pollution owing to its high microwave absorption properties [45]. rGO leads to better attenuation performance of nanocomposites while increasing dielectric loss, thereby improving the entry and absorption of microwaves by the composites (Figure 4). Despite the low specific capacitance of rGO, when used in tandem with selenium, the specific capacitance can be increased to $390 \mathrm{~F} \mathrm{~g}^{-1}$, enabling the use of these composites in supercapacitors [46]. As GO displays properties of transparent conducting oxides, it can be used as a semiconductor, particularly due to its flexibility in deposition [47].

The potential for widespread application of graphene is easy to predict, particularly considering its wide range

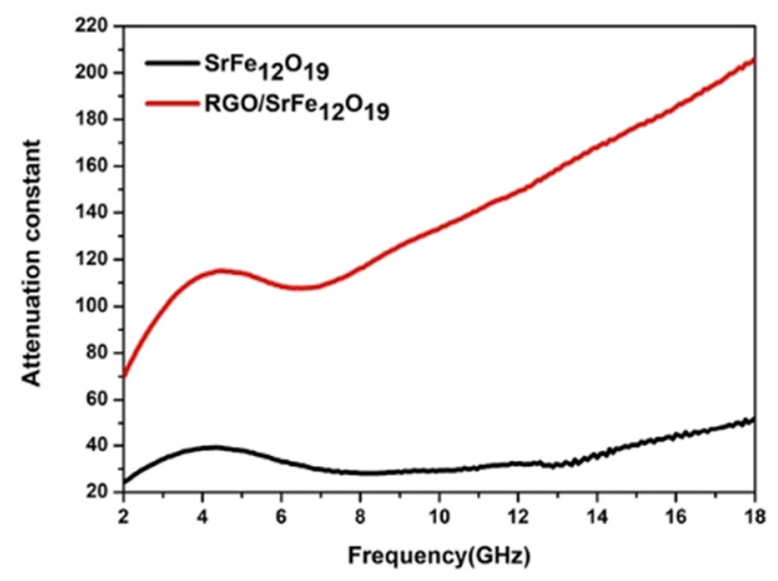

Figure 4: Attenuation constants of nanocomposites with changing frequency [45]. 
of functional properties. Research into the commercial synthesis of single-layer graphene is still ongoing, which focuses on improving the quality and scalability [48]. As a result, efficient synthesis and appropriate starting materials need to be identified before this can be realized at an industrial level. Thus, this review aims to evaluate the suitability of several starting materials for the synthesis of graphene by considering the quality of graphene produced and its use in various applications. The most effective synthesis methods associated with these precursors are also discussed by considering the end product and any complications related to the processing steps. As aforementioned, this research into alternative starting materials will place less strain on the finite global graphene resources and allows the production of graphene with more functionality, enabling its use in a wider array of applications. Low-cost graphene will also become a possibility through the use of inexpensive or discarded materials allowing the widespread use of graphene. Identifying appropriate synthesis methods for each precursor in large-scale production of graphene is expected to help bloom the graphene industry and increase its widespread availability.

\section{Synthesis of graphene}

Graphene synthesis methods are generally classified into two categories: top-down and bottom-up approaches, as detailed in Figure 5 [49].

\subsection{Top-down}

The top-down method is a destructive technique by which large starting materials are broken down by delaminating graphitic layers into graphene [50]. Two general top-down methods are exfoliation and reduction [51], using mechanical, chemical, thermal, or electrochemical means. Some chemical reactions are known to take place during the synthesis process. In the mechanochemical ball-milling method, the kinetic energy of grinding media is used to unzip layers of graphite and cause chemical reactions to occur at the unzipped edges, thereby functionalizing the graphene leading to self-repulsion [52]. In the top-down approach utilizing solar radiation, there is a high-temperature chemical reaction between the graphite surface and the surrounding water, resulting in the exfoliation of GO to graphene [53]. While relatively easier to scale up, the top-down approach presents several challenges as graphene synthesized by these means display inconsistent properties, namely, low production yields and poor conductivity [54].

\subsubsection{Mechanical exfoliation}

Mechanical exfoliation uses directional force, either normal or lateral, to peel layers of graphene from bulk graphite by overcoming the van der Waals forces of attraction between the layers [55]. The process requires a starting graphite material such as highly oriented pyrolytic graphite (HOPG), single-crystal graphite, or natural graphite [56] and an applied force of around $300 \mathrm{nN} \mu \mathrm{m}^{-2}$ [57]. The typical mechanical exfoliation techniques include micromechanical cleavage, continuous mechanical cleavage, shear exfoliation, and explosive exfoliation. While all these methods utilize mechanical force, the equipment by which this force is exerted varies from mills, blenders to tape. These methods are also attempted at relatively low processing temperatures apart from the explosive technique, which requires high energy for detonation.

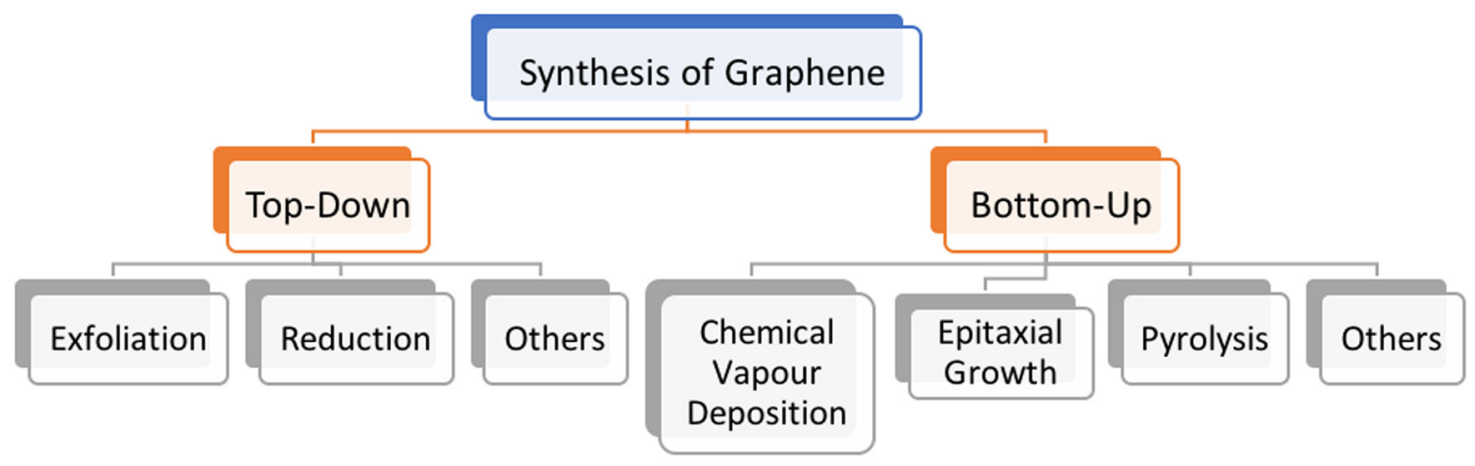

Figure 5: Methods followed for the synthesis of graphene. 


\subsubsection{Micromechanical cleavage}

As the first established method for the extraction of graphene flake [3], the Scotch tape method begins by cleaving the top few layers of bulk graphite using the adhesive tape. This starting material is prepared by compressing HOPG mesa against a 1-mm-thick photoresist layer over a $300-\mathrm{nm}$-thick silica $\left(\mathrm{SiO}_{2}\right)$ substrate which is further secured through the process of baking. As the adhesion of graphene to the $\mathrm{SiO}_{2}$ substrate is stronger than the van der Waals forces of attraction between the graphene layers, these layers are transferred to the substrate surface on the removal of the tape by the normal force. This process is repeated several times, thinning out the graphene layer, until a monolayer is obtained. Theoretically, this method works with any substrate with good adhesion to graphene, such as aminotriazine [58].

Before the advent of this method, mechanical exfoliation was performed using the tip of atomic force microscopy, which enabled the production of $200 \mathrm{~nm}$ thick multilayered graphite. On the other hand, the Scotch tape method can isolate a single graphene layer, less than $10 \mathrm{~nm}$ thick, from a $1 \mathrm{~mm}$ thick graphite layer [59]. The scotch tape exfoliation method was recently modified to produce graphene nanosheets using graphene quantum dots [60]. This sonication-assisted process can increase the yield of nanosheets from $40 \%$ [61] to almost $100 \%$ without introducing structural defects on the basal plane [60], allowing for better electrical conductivity. The process also imparts long-term stability of graphene in water due to the electrostatic repulsion contributed by the graphene quantum dots.

While the scotch tape method yields high-quality graphene with very few defects, the production rates possible through this process remain very low and only suitable for fundamental scientific testing [62]. The repeated peelings are labour-intensive and challenging to mechanize due to difficulties in predicting the required number of peelings. Micromechanical cleavage was successfully scaled up to a lathe-like set-up utilizing an ultra-sharp single-crystal diamond wedge to produce graphene flakes from bulk graphite [63]. The durability of the diamond wedge and the reproducibility of this method make it a viable low-cost alternative; however, the effects of operating conditions on the quality of graphene need to be understood before industrial scale-up can be realized.

\subsubsection{Continuous mechanical cleavage}

The Scotch tape method inspired another method [64] for the synthesis of monolayer and few-layer graphene (FLG) from natural graphite using a three-roll-mill machine with a polymer adhesive, usually polyvinyl chloride (PVC). In this method, the graphite and polymer adhesives are placed between moving rolls causing exfoliation by shear force to form graphene. Similar to the use of a three-roll-mill machine, Liu et al. [65] and León et al. [66] also successfully synthesized graphene using a ball mill with ammonia borane and melamine, respectively. Graphene layers are as thin as $1.13 \mathrm{~nm}$ [67], and the adhesive costs are too high to justify industrial use, especially considering graphene that has to be purified of the adhesive. The removal of PVC requires $5 \mathrm{~h}$ of heating at $500^{\circ} \mathrm{C}[64]$, which is energy intensive and contributes to the production cost. More studies are needed to further understand the mechanisms, thereby a solution to the issues associated with adhesive use could be identified.

\subsubsection{Shear exfoliation}

Graphene exfoliation can also be performed by adding a shear force to a precursor, generally graphite, in a stabilizing liquid such as sodium cholate. Unlike in liquidphase exfoliation (LPE) synthesis, this method does not require sonication and produces defect-free and unoxidized graphene [68]. The use of sonication in a synthesis method casts doubts onto its commercial application as the scale-up of a sonication process is notoriously challenging. This synthesis method has proved successful even when using a simple kitchen blending which achieves shear rates higher than $10^{-4} \mathrm{~s}^{-1}$ [69], suggesting that the scale-up is relatively a simple process. However, the exfoliation yield of this method is as low as $0.1 \%$ even at extremely high shear rates. The recycling of residual graphitic sediment has been proposed to alleviate this problem, yet this only increases the exfoliation yields to at least $3 \%$. Further study into shear exfoliation in aqueous solvents, as opposed to organic solvents, is needed, as this would increase the inherent scalability of the system. A system for controlling the solution temperature, such as modifying the blender for fans [70], may prove useful as a significant amount of heat is generated in the blending process. Graphene produced through this method is recommended to reinforce meltprocessed composites and the production of highly conductive nanosheets for electrode applications.

\subsubsection{Explosive exfoliation}

The controlled detonation of acetylene in the presence of oxygen can produce graphene nanosheets in a combustion 
chamber [71]. During detonation, acetylene is converted into free carbon atoms, which condense into carbon nanoparticles upon cooling the combustion chamber. These nanoparticles then aggregate to form graphene with a yield per detonation as high as $66 \%$. This process does not produce carbonaceous soot, as the detonation temperature of $4,000 \mathrm{~K}$ is twice the combustion temperature for soot production. The study also claimed simple modifications made to the apparatus, which could produce up to $300 \mathrm{~g} / \mathrm{h}$ of graphene, showing tremendous potential for scale-up. Explosions can also be generated by applying an electric wire explosion charge voltage on a pure graphite stick in water under ambient conditions [72]. Energy injected into the system through this explosion overcomes the van der Waals forces, thereby breaking the graphite into graphene of less than 10 layers thick. A mechanism for controlling the explosion force needs to be devised; however, the uncontrolled explosions can lead to a complete disruption of graphene crystallinity. While graphene synthesis through explosion is an extremely rapid and high-yield process, several safety issues are associated with it. Extremely rigorous control and fine-tuning of the processing parameters are required not only because they significantly affect the quality of resultant graphene but also because this can lead to runaway explosions.

\subsubsection{Chemical exfoliation}

Chemical exfoliation utilizes a liquid suspension that converts graphite to graphene by the formation of graphene-intercalated compounds. Alkali metals are used to increase the interlayer spacing by reducing the van der Waals forces of attraction between graphene layers owing to the potential ionization differences between these alkali metals and graphite [73]. While chemical exfoliation has the advantage of high yield and low operating temperature, the graphene produced through this strategy tends to reassemble back into graphite, necessitating the need for surfactants or immiscible liquids [74]. Typical chemical exfoliation methods include LPE and supercritical fluid exfoliation. Both techniques require the use of a solvent through which the graphene is dispersed at relatively low operating temperatures. LPE uses sonication to overcome the forces of attraction between graphene layers, and supercritical fluid exfoliation functions through the penetration of the fluid to separate the graphene layers.

\subsubsection{LPE}

LPE generally starts with the dispersion of graphite in a suitable solvent, followed by sonication-induced exfoliation occasionally in the presence of surfactants. Under sonication, shear forces and cavitation (the growth and collapse of bubbles due to pressure fluctuations) act on the bulk graphite to overcome the van der Waals forces between the graphene interlayers, which trigger gradual exfoliation into smaller flakes. It is important to note that solvent molecules by themselves cannot inherently dissolve graphene, and solvent-graphene interactions thus need to be equivalent to intersheet attractions of graphene after exfoliation to prevent their restacking. However, this solvent-graphene interaction alone is usually not strong enough to suppress the van der Waals forces between graphene interlayers. To combat this, the addition of surfactants to the solvents promotes the exfoliation of graphite and long-term stabilization of graphene suspensions, as the molecular adsorption of surfactants onto the basal plane of graphene has higher energy than the solvent-graphene interactions. Besides surfactants, polymers such as polybutadiene, polystyrene-co-butadiene, polystyrene (PS), PVC, polyvinyl acetate, polycarbonate, polymethyl methacrylate (PMMA), polyvinylidene chloride, cellulose acetate, ethyl cellulose, and polyvinylpyrrolidone can also be appropriately used in LPE [75]. Although this process produces good quality graphene with lower costs than the time-consuming processes such as the Scotch tape method, very little is understood about the fragmentation process. Only recently, the statistical models [76] have begun unveiling the mechanisms behind this synthesis, suggesting that erosion functions in tandem with peeling. More studies need to be performed to determine the stages by which this synthesis works to identify the sources of defects or surface roughness and whether it is feasible to functionalize the produced graphene.

\subsubsection{Supercritical fluid exfoliation}

Graphene sheets can also be produced by intercalating graphite with supercritical fluids, which are expanded to push the graphene layers apart. First, graphite is immersed in a supercritical fluid, most commonly supercritical $\mathrm{CO}_{2}$. Subsequently, the supercritical $\mathrm{CO}_{2}$ penetrates into the interlayers of graphite with the help of ultrasonication. Following this, the rapid depressurization of supercritical 
$\mathrm{CO}_{2}$ causes $\mathrm{CO}_{2}$ to expand and separate the graphite layers to form monolayer graphene or FLG sheets. To avoid restacking, the graphene sheets are then collected by discharging the expanding $\mathrm{CO}_{2}$ gas directly into a solution with sodium dodecyl sulphate [77]. This method provides a rapid and easily scalable production avenue for the synthesis of pristine graphene [78] and contributes to the added benefits of utilizing an environmentally friendly exfoliation medium of supercritical $\mathrm{CO}_{2}$. The efficiency of this technique can be enhanced by incorporating ultrasonication and applying shear stress.

GO composites have also been produced using supercritical sodium tetraborate decahydrate under relatively low temperatures of $400^{\circ} \mathrm{C}$ [79]. This results in the reduction of $\mathrm{GO}$ and allows for the boron doping of graphene to provide added functionality to the graphene. Supercritical acetone has recently been used to synthesize reduced $\mathrm{GO}$ in $5 \mathrm{~min}$ at $400^{\circ} \mathrm{C}$ [80], similar to the study mentioned above. It is suggested that supercritical alcohols [81] can also be appropriate for the synthesis of graphene.

Supercritical processing is much favoured in nanomaterial synthesis due to its short reaction times compared to the thermal exfoliation techniques. However, the success of this method is significantly dependant on the employed supercritical fluid with variations seen in the quality and functionality of the graphene or GO produced. While the energy demands are low due to the low reaction temperatures, the environmental impact of the process also relies on the utilized supercritical fluid.

\subsubsection{Electrochemical exfoliation}

The electrochemical set-up used to exfoliate graphite usually contains key elements: a graphite working electrode, counter electrode, reference electrode, electrolyte, and power supply. HOPG, graphite powders, graphite rods, graphite foil, or graphite flakes are the typical working electrodes [82]. Platinum wire, mesh, plates, or rods, and graphites are most frequently used as counter electrodes. The mechanisms behind electrochemical exfoliation principally depend on the type of potential applied, i.e. anodic or cathodic. Anodic exfoliation involves the intercalation of anions and any co-intercalating species in the reaction mixture into graphite. A positive current withdraws electrons from the graphite working anode, creating a positive charge. This charge encourages the intercalation of bulky negative ions, such as sulphate anions $\left(\mathrm{SO}_{4}{ }^{-}\right)$, which increase the interlayer spacing between graphene sheets and assist in the subsequent exfoliation of the sheets. In cathodic exfoliation, a negative bias at the graphite working electrode attracts positively charged ions in the solution (e.g. $\mathrm{Li}^{+}$), along with any co-intercalating molecules. Again, the wedges of these intercalating species open the graphene sheets, causing expansion and exfoliation [79]. The graphene produced through this technique has minimal defects and can be used in electronics, energy storage devices, and nanocomposites. The electrochemical exfoliation has lower mass production costs [76] and does not require the use of harsh chemicals, and electrochemical activation negates the need for additional purification steps. This promising process uses a "one-pot approach," and the level of oxidation of the resulting graphene is tuneable. A variant of electrochemical exfoliation, the electric spark discharge method [83], allows varying the pulse cycle switching times in order to manipulate the suspension properties of the graphene. There is still a major limitation to this synthesis as supplying an unbroken voltage bias to the graphite is difficult, and failure leads to irregular exfoliation, which becomes even more pronounced during scale-up owing to the larger areas of exposed graphite edges [84]. The system needs to be redesigned to allow for the efficient application of the electrochemical driving force to the graphite.

\subsubsection{Chemical reduction}

The chemical composition of GO does not differ greatly from that of graphite oxide as it is only structurally different in terms of the number of stacked graphene layers. Hence, the followed reduction of graphite oxide can also be applied to GO. GO is usually immersed in a chemical reducing agent at a particular temperature range for a while [85], and the reducing agent transforms the brown GO to black graphene. Besides the noticeable colour change, the increase in hydrophobicity or aggregation of graphene after the removal of oxygen-containing groups indicate the completion of the reaction. Numerous chemical reducing agents are available for $\mathrm{GO}$, and the reaction mechanisms of which have not been elucidated. These have not been previously applied in synthetic chemistry as reducing agents and do not have any definite modes of reaction towards specific oxygen functional groups [86]. While the chemical reduction can produce high-quality graphene by removing most of the functional groups associated with GO, it frequently makes use of hydrazine as a reducing agent that is both costly and toxic. While research is still ongoing to find a more 
acceptable alternative, large-scale adoption of these reducing agents is hindered by long synthesis times and chemical costs.

\subsubsection{Electrochemical reduction}

The electrochemical reduction of GO is usually carried out via two different routes, namely, one-step and two-step approaches. In the one-step electrochemical approach, GO is electrochemically reduced to $\mathrm{rGO}$ (ERGO) thin films in an aqueous colloidal suspension using a buffer electrolyte. The electrochemical reduction process can be performed with cyclic voltammetry [87], linear sweep voltammetry [88], or at a constant potential mode in a standard three-electrode electrochemical system at room temperature. The electrochemical reduction is believed to occur when the GO sheets adjacent to an electrode accept electrons, yielding insoluble ERGOs that attach directly onto the electrode surface. Using this approach, ERGOs were successfully harvested onto a glassy carbon surface [89], which acts as the substrate, yet any conducting surface can be used in its place. Graphene produced in this way is highly stable due to its low solubility in common solvents. A GO thin film is first deposited on the electrode substrate in the two-step electrochemical approach, forming a GO-coated electrode after a drying process. The GO-coated substrate electrode can then be electrochemically reduced using a standard three-electrode electrochemical system with a buffer or supporting electrolyte, synthesizing ERGO films on the electrode substrate [90]. The properties of ERGOs differ from pristine graphene due to the presence of residual oxygen functionalities on the carbon basal plane [91] while still possessing the graphene structures. These properties can be easily manipulated by varying the electrolysis parameters and the electrolyte [92]. The electrochemical reduction is a fast synthesis method that is economically viable and environmentally friendly, as it does not involve the use of toxic reductants usually associated with chemical reduction. Unlike electrochemical exfoliation, which preserves the quality of graphene, the electrochemical reduction restores some of the original properties and exploits new functionalities of the rGO [93]. However, as the reduction rate is so rapid compared to chemical reduction, several defects formed are not removed as the reduction time increases [94]. This indicates that further processing, such as annealing the products or performing the reduction at higher temperatures, is required.

\subsubsection{Thermal exfoliation reduction}

Graphite with interlayer functional groups such as graphite oxide, expanded graphite, and intercalated graphite compounds are usually used as the starting materials for the thermal exfoliation reduction. These materials are heated up to their respective decomposition temperatures and the functional groups on the graphitic layers then decompose to produce gases that build up pressure between them. Thermal exfoliation reduction occurs when this pressure exceeds the van der Waals forces between these interlayers. Hence, it is essential to choose materials with interlayer functional groups as the starting materials instead of pure graphite to ensure the build-up of pressure required for exfoliation [95]. A recent study showed that $\mathrm{GO}$ reduction first occurs at $127^{\circ} \mathrm{C}$, continues smoothly above $600^{\circ} \mathrm{C}$ with the loss of oxygen and hydrogen. In the process, the hybridized carbons are converted from $\mathrm{sp}^{3}$ into $\mathrm{sp}^{2}$, confirming the reduction process. The critical temperature appears to be $1,000^{\circ} \mathrm{C}$ for $\mathrm{GO}$ reduction, where the water molecules and oxygen functional groups are evaporated and burnt, and the resultant graphene contains $<2 \%$ oxygen and $81.5 \% \mathrm{sp}^{2}$-carbon atoms [96]. While thermal exfoliation reduction has proven to be a very efficient method for preparing graphene, the sheets produced exhibit a wrinkled and defective structure [95]. As this process involves burning, a significant amount of $\mathrm{CO}_{2}$ is produced, contributing to greenhouse gas generation. Furthermore, the high temperatures associated with this process increase the manufacturing costs and the need for rigorous control of operating conditions [97].

\subsection{Bottom-up}

The bottom-up method is the assembly of graphene from smaller carbonaceous elements onto a substrate [98]. Graphene can be synthesized using several bottom-up methods, namely, chemical vapour deposition (CVD), epitaxial growth and pyrolysis, etc. [51]. All these require the precursors in gaseous form to allow for the deposition onto a substrate. CVD typically requires a vacuum, ensuring that the reactants reach the substrate without interference. Still vacuum condition is not necessary for epitaxial growth and pyrolysis, indicating lower manufacturing costs. The substrates for each method differ with CVD requiring a transition metal substrate, and 
spray pyrolysis utilizing a glass substrate, but epitaxial growth makes use of silicon carbide (SiC).

\subsubsection{CVD}

CVD is the deposition of gaseous reactants onto a substrate to form graphene. First, a gas-phase carbon precursor is adsorbed onto the surface of a transition metal catalyst, which undergoes catalytic decomposition to carbon atoms. Then the decomposed carbon species diffuse and dissolute into the metal at elevated temperatures. The dissolved carbon atoms are then precipitated out and segregated onto the metal surface. On cooling, surface nucleation initiates, followed by the graphene growth [99]. The resulting graphene quality is mainly determined by the processing parameters such as catalysts, precursors, gas flow rate, temperature, pressure, and time. Transition metals with an incomplete shell (e.g. Ni and $\mathrm{Co}$ ) exhibit a certain degree of carbon solubility and thus produce a few-layer polycrystalline graphene with a larger area. On the other hand, transition metals with filled shells (e.g. $\mathrm{Cu}$ and $\mathrm{Zn}$ ) possess low affinity to carbon, and hence both dissolution and subsequent segregation steps are not possible. In this case, carbon atoms derived from hydrocarbon precursors could directly diffuse onto the metal surface and build up thermodynamically stable graphene. This process easily results in the formation of large-area monolayer graphene. Over $95 \%$ of monolayer graphene films could be achieved using binary $\mathrm{Ni}-\mathrm{Cu}$ alloys as the catalytic substrates [100].

CVD leads to very-high-quality, large-area, and single crystal graphene and is thus frequently the synthesis method of choice. More recently, the cold-wall CVD method has attracted interest over the conventional approach featuring a less complicated reactor, short deposition times, and rapid heating and cooling rates, which is instrumental in achieving fast graphene growth. In this technique, the substrate and the chamber walls remain at room temperature, negating heating the entire chamber. The cold-wall method can suppress the gasphase reactions associated with high-temperature graphene growth resulting in the production of super clean graphene [101]. This reactor can also be appropriated for plasma-enhanced CVD. Microwave-plasma-enhanced CVD is carried out at reduced temperatures of $760^{\circ} \mathrm{C}$, which can achieve rapid processing in a single step [102]. Current-enhanced CVD utilizing nickel catalysts can produce high-crystallinity multilayer graphene at a low temperature of $464^{\circ} \mathrm{C}$ [103]. The dimensions of the synthesized graphene are not constrained to the size of the initial bulk graphite, allowing polycrystalline singlelayer graphene to be made as large as the underlying substrate. The quality of the graphene produced is sensitive for electronic applications, as the graphene grows in a self-limiting way to a single or few layers only. The graphene produced is easily transferred from the metal surface to an arbitrary substrate by supporting the graphene with a polymer and etching away the metal. However, the transfer may contaminate and damage the graphene sheet. The synthesis cost of graphene using the CVD method is moderate, as it is cheaper than the cost of epitaxial growth but more expensive than the cost of reducing GO [5].

\subsubsection{Epitaxial growth}

Epitaxial growth of graphene typically uses $\mathrm{SiC}$ as the starting material. First, $\mathrm{SiC}$ is heated to a high temperature under vacuum conditions where it undergoes thermal decomposition and Si sublimates. Then the decomposed $\mathrm{SiC}$ undergoes an annealing process, which involves gradual cooling after heating. This allows the sublimated $\mathrm{Si}$ to be reconstructed as an interfacial layer on $\mathrm{SiC}$, and the $\mathrm{C}$ deposits on the $\mathrm{Si}$ face as an epitaxial layer to produce graphene. However, Si may still evaporate during the deposition of $\mathrm{C}$. To overcome this problem, vacuum condition is substituted by introducing argon gas atmosphere at near ambient pressure, which successfully hinders the transport of $\mathrm{Si}$ atoms away from the $\mathrm{SiC}$ surface. This reduces the overall sublimation rate and increases the graphitization temperature by several hundred degrees [104]. This technique has good control over the number of graphene layers formed, as the heating temperature is regulated to produce graphene with promising electronic properties. However, the homogeneity of graphene produced through this energy-intensive method is rarely observed. The graphene quality could further degrade by the development of a C-polar face rather than the S-polar face that facilitates homogeneous growth of graphene [105].

\subsubsection{Spray pyrolysis}

Spray pyrolysis involves the spraying of graphene dispersion onto a heated substrate to obtain a thin film of graphene. The graphene dispersion is first pumped into a nebulizer and a carrier gas, which is usually compressed air. The venturi effect created by the compressed air 
flowing through the jet enables the solution to be nebulized. The baffle placed at the tip of the jet produces fine aerosol droplets while blocking and returning the larger ones into the reservoir, yielding a stream of uniform and fine droplets. These uniform and fine droplets are then sprayed onto a heated glass substrate to form the graphene films [106]. The advantage of the spray pyrolysis method is that thin and homogeneous graphene film can be produced. Additionally, no high temperatures are required as the glass substrate only needs to be heated up to $200^{\circ} \mathrm{C}$. As the reduction occurs thermally, reducing agents are not required. Moreover, vacuum condition is not required, and hence the cost to generate a vacuum environment is avoided. The only utility cost to account for in this method is the compressed air (1 bar), increasing the production cost slightly [106].

\section{Graphene precursors}

Several precursors have been used to synthesize graphene, as displayed in Figure 6.

\subsection{Conventional precursors}

\subsubsection{Graphite}

Graphite is a common precursor to graphene (a single layer of graphite), which is the most widely studied raw material for the synthesis of graphene. Still, the argument is that any of the crystalline phases of graphite, notwithstanding the stacking arrangement, comprise the structural unit "graphene layer" [107]. Thus, thickness becomes a vital parameter in the synthesis of graphene from graphite to establish when a material can reasonably be called graphene. When the number of graphene layers in a material exceeds ten [108], it becomes impossible to differentiate its electronic structure from graphite, allowing this number of layers to be widely accepted as the boundary between graphite and graphene materials. While not a perfect threshold, as it only considers the variations in the electronic property, this number still provides some physical basis by which these materials could be classified. The close association between these materials leads to several established processes for graphene generation such as micromechanical exfoliation, mechanical cleavage, LPE, supercritical fluid, and electrochemical exfoliation.
Micromechanical exfoliation presents the advantage of being a relatively inexpensive and straightforward process, as the key materials are bulk graphite, a substrate, and adhesive tape [3], yet the graphene produced is of high structural and electronic quality. However, it is a time-consuming process and thus too inefficient for mass production. A large amount of debris is produced, which burdens the industrial setting with sensitive equipment and imposes high contamination risks [109]. Its low yield is also a limitation and hence considered for scientific studies only. Furthermore, the dimensions of the produced graphene are constrained to the size of the starting material graphite [5]. To increase the yield, a rigid or viscous substrate has been used [109].

Continuous mechanical cleavage is an industrially applicable method for the production of graphene from bulk graphite; however, the obtained graphene is a mixture of monolayer graphene and FLG as well as un-exfoliated graphite flakes, which reduce the quality and grade of the product [64]. It has been suggested that ball mill, which is common in the powder production industry, produces graphene of better quality with five layers [56] or less than ten layers [64] or less obtained by the conventional three-roll-mill technique. While FLG is produced, the numerous defects, especially basal plane defects caused by the collisions of grinding media, severely limit the application of this graphite-derived graphene. A similar issue is seen when graphite is electrochemically exfoliated as the produced graphene might be slightly oxidized with inhomogeneous thickness [110,111].

Similarly, the LPE method applies sonication to graphite for long periods at high powers which increases the dispersibility of graphene, as reported by Skaltsas et al. [112], but the harsh conditions invoked by cavitation damage the graphene. Besides the defects found in the produced graphene, issues are also associated with the solvents used for LPE. For example, $N$-methylpyrrolidone and $\mathrm{N}, \mathrm{N}$-dimethylformamide are reproductive toxicants, whereas 1,2-dichlorobenzene demonstrates high aquatic toxicity and thus pose health and environmental risks if the graphene derived from this graphite is used in biological applications.

Graphite can also be subjected to supercritical fluid exfoliation to produce monolayer and bilayer graphenes of high yield. Still, the electronic properties of graphene are severely affected with the introduction of this surfactant as the sulfonic acid groups in 1-pyrene sulfonic acid sodium salt (1-PSA) act as electron-withdrawing groups resulting in electron transfer from graphene to 1-PSA molecules. From these studies, it is clear that the quality of graphene produced from graphite is greatly dependant 


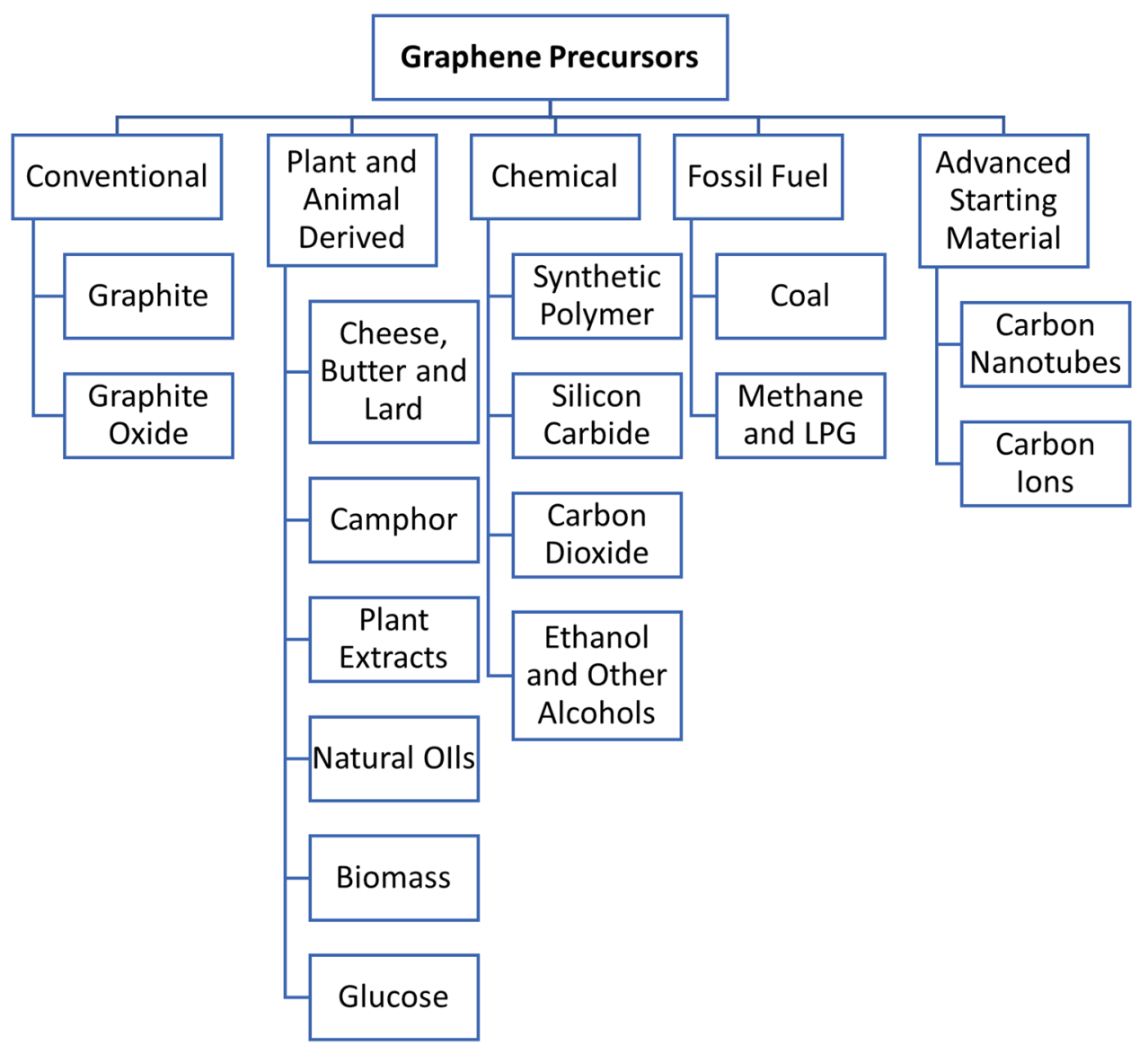

Figure 6: Potential precursors utilized for the generation of graphene.

on the processing method used. While capable of producing high-quality graphene in some instances, the overuse of graphene as a precursor has to be addressed.

The world's graphite stores are not as bountiful as other materials such as coal, and thus the limitation in supply makes high-purity graphite expensive and difficult to source [113]. The synthetic production of graphite is currently complex and requires several raw materials as the mechanism of interaction between graphite crystallites and porosity is still not fully understood [114]. The extraction of graphite from waste products, such as discarded dry cell batteries, is an environmentally friendly and cost-effective source for graphene production. Previous studies succeeded in synthesizing graphene from waste battery graphite electrodes through Hummers' method [115] and electrochemical exfoliation [116]. Bandi et al. [117] increased the efficiency of the process by utilizing both the graphite anode and the cathode in place of inert materials, which led to a GO yield of $88 \%$.
The wealth of information provided by extensive studies of graphene makes it an attractive starting material. As a non-renewable resource with already limited reserves, the consequences of extensive use of graphite must be considered with the necessity for more studies into the possibility of graphite reuse from waste for the production of graphene. While most graphite processing methods show high graphene yields, the surface defects are extensive, mainly due to the agglomeration rates. Hence, appropriate non-polluting and non-toxic surfactants and stabilizers need to be identified to overcome this problem further to establish graphite as a viable industrial starting material.

\subsubsection{GO}

GO is a hydrophilic, non-conducting carbon material with a graphene lattice structure dotted with several 
functional groups including alcohols, ketone carbonyls, and carboxylic groups. GO is of particular interest in the generation of adsorbents as it allows excellent functionalization, thereby resulting in chemically modified graphene, which can adsorb specific substances such as pollutants from wastewater.

Graphene is conventionally produced by the chemical reduction of GO; however, this product is prone to aggregation and still shows defects as the remaining functional groups cause disorder in the electronic structure of the graphene leading to reduced electrical conductivity [118]. Alternatively, the electrochemical reduction of graphite can be used to produce graphene free from contamination arising from the residue during the reduction. These contaminants severely affect and alter the unique properties of graphene. The electrochemical approach can produce graphene directly onto electrode substrates which can be used for specific applications, such as in biosensors [119] and electrocatalysis [120] without any further steps or treatments [90]. This method yields verythin-layer graphene nanosheets with flake-like shapes, wrinkles, and good transparency (Figure 7).

GO needs to be oxidized and reduced before it can be used for the production of graphene monolayer to separate and isolate the layers without modifying their structure. Although all the previously described methods can be scaled up for commercialization, the produced graphene is still of significantly inferior quality to that produced through mechanical exfoliation. The abundant use of toxic chemicals such as $\mathrm{HNO}_{3}$ and $\mathrm{KClO}_{3}$ in graphene synthesis with GO hinders widespread adoption of these synthesis methods. Hence, more studies are necessary to identify safer methods. GO from pure graphite can be easily synthesized on-site to keep the manufacturing

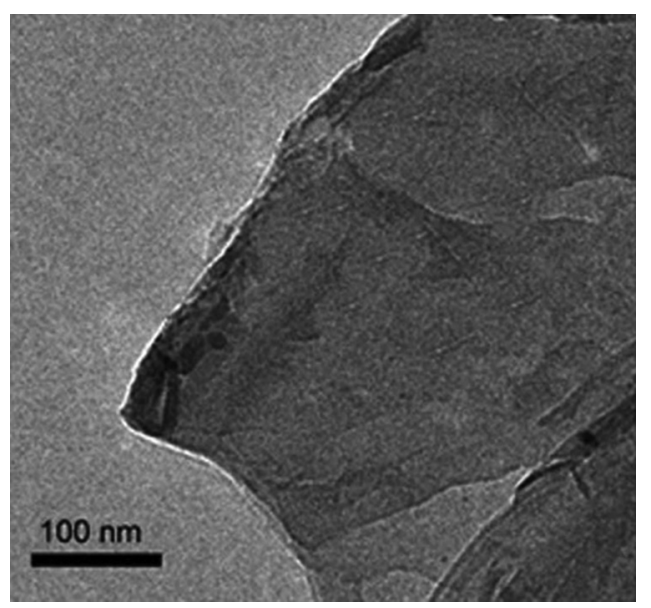

Figure 7: TEM image of methacrylated GO for biosensors [119]. costs low. Most commercially available GO is of small size as graphite oxidation requires graphite particles to be smaller than $10 \mu \mathrm{m}$ owing to intense stirring and ultrasonication. However, to maintain the structural integrity during graphene synthesis, large flakes are preferable. GO was also prepared in two stages, soft chemical exfoliation followed by oxidation, an effective method in producing large and ultra large-sized GO platelets without oxidation defects [121]. These advances in improving the quality of GO obtained through graphene will be vital if $\mathrm{GO}$ is adopted on a commercial scale.

\subsection{Plant- and animal-derived precursors}

\subsubsection{Cheese, butter, and lard}

Vertical graphene nanosheets (VGNs) have excellent electrical transport properties, large surface areas, and, most importantly, an inherent three-dimensional, open network structure. Thus, VGNs hold great promise for high-performance supercapacitors. However, it remains challenging to materialize VGN-based supercapacitors due to their low specific capacitance, high temperatures for processing, poor binding to electrode support materials, uncontrollable microstructure, and non-cost-effective fabrication method [122,123].

Recently, Seo et al. successfully transformed cheese [122] and butter [123,124] into VGNs within few minutes through treatment with argon and hydrogen plasma without catalyst or external heating. These VGNs processed at low temperatures were reported to exhibit reliable biosensing properties, strong binding with proteins, and improved adhesion to substrates [122,124]. The VGNs derived from cheese and butter showed high areal capacitance of $0.46 \mathrm{~F} \mathrm{~cm}^{-2}$ [122] and high specific capacitance up to $230 \mathrm{~F} \mathrm{~g}^{-1}$ [123]. The resulting VGNs displayed sharp, open edges similar to the graphene synthesized from a hydrocarbon gas. These properties demonstrated that cheese- and butter-derived VGNs might be directly employed as binder-free supercapacitor electrodes [123]. Hence, VGNs may be the promising materials as advanced energy storage devices [123] in electronic and energy applications [122].

Waste lard oil is even cheaper and more environmentally friendly than cheese or butter, as it can be sourced from cooking waste and be transformed into value-added products. Waste lard oil acted as a carbon donor in the electrochemical synthesis of VGNs using inductively coupled plasma-enhanced CVD [125]. However, high 
temperatures of up to $800^{\circ} \mathrm{C}$ were required, as carbon clusters are observed at lower temperatures. Yet temperatures exceeding $900^{\circ} \mathrm{C}$ led to extremely dense, lowquality graphene due to the surface reaction kinetics causing the growth of graphene. The VGNs produced from waste lard oil were also superhydrophobic with a contact angle of more than $140^{\circ}$.

Cheese and butter are non-polluting and non-toxic materials that can produce VGNs with the admirable quality of high specific capacitance. Nevertheless, these two precursors need to be processed before graphene synthesis. Cheese contains large quantities of water, fats, and proteins, thus requiring a dehydration process. Plasma heating at low pressure can remove water and break down the fats and proteins into simple hydrocarbon units [122]. The synthesis can be performed at low temperatures $\left(400^{\circ} \mathrm{C}\right)$ and hence requires less energy. However, the extensive use of cheese and butter brings up the food vs fuel dilemma [126] wherein the risk of diverting such food to product synthesis such as graphene may come as a detriment to the food supply as the appropriate allocation of resources must be considered. Waste lard oil may be a solution to this issue, as it can no longer be consumed as food, and there should be no qualms about using it for the synthesis of graphene. The precise temperature control in this system is very rigorous and necessary to obtain satisfactory VGNs that necessitate constant monitoring. Further testing is required to determine whether VGNs produced in this way is economically feasible as significant funds will need to be allocated for process monitoring and controlling.

\subsubsection{Camphor}

Camphor is obtained from the camphor laurel tree, which is frequently used as a plasticizer. As it is an inexpensive carbon compound, its role as a starting material for graphene synthesis should be considered. Camphor is also non-toxic, non-polluting, and renewable resource [127].

Ravani et al. [127] synthesized graphene from camphor using the CVD method but controlled the thickness of the graphene film by allowing the system to cool down to room temperature on its own rather than rapid cooling. The process temperature of this method was remarkably reduced from the normal CVD temperature of 1,000 to $850^{\circ} \mathrm{C}$, making it a more energy-efficient process. Some wrinkling of the graphene films caused by the variations in the thermal expansion between the substrate and graphene and some defects in the form of carbon structures were also found as a result of condensation of camphor upon cooling. The graphene films were also not uniform with thicker regions found in some of the sections. A similar low-temperature CVD method resulted in the growth of a continuous layer of camphor-derived graphene on a copper substrate at $800^{\circ} \mathrm{C}$ [128]. The threezone CVD system allows controlled heating, which enables the formation of a uniform graphene layer. These systems are significantly more energy efficient than conventional graphite processing methods, and the graphene produced is of high enough quality to enable its use in the optoelectronic industry.

Recently, camphor-derived graphene sheets were synthesized via atmospheric CVD processes on various films [129]. The film produced was uniform and appreciable electrical and optical properties with a maximum transmittance of $80 \%$ at $550 \mathrm{~nm}$. The nucleation and growth of the graphene crystals could be controlled by varying the annealing and gaseous flow rates [130]. However, the post-growth processing was time-consuming, with etching taking several hours, after which the substrates need to be stored for extended periods to allow complete adhesion to the substrate. At high camphor concentrations, several defects were seen in the produced graphene which may be due to an increase in the number of nucleation sites available for graphene crystals, which can form stacks of graphene layers [129].

Thus, camphor-derived graphene can be produced through relatively inexpensive methods, either under atmospheric conditions or at lower temperatures. The hydrocarbon gas precursors in the usual CVD methods can be replaced by camphor, an abundant and inexpensive natural precursor. Camphor-derived graphene can be used in electronic [131] or optoelectronic [132,133] applications where graphene films have to be transferred to glass and plastic substrates to act as transparent electrodes. Transparent camphor-derived graphene films can be used in solar cell applications [134].

\subsubsection{Plant extracts}

Using plant extracts is a means of producing "green" graphene, as conventional carbon precursors are originated from non-renewable fossil fuels. However, complex distillation and purification processes can greatly increase the cost of these extracts, which causes graphene synthesis from these precursors uneconomical. The simplicity of the extraction process varies among plants depending on their active ingredients; thus, the nature of the plants from which these extracts originate must also be considered. 
Jacob et al. [134] synthesized graphene films using the volatile natural extract of a tea tree, Melaleuca alternifolia, through a plasma-assisted CVD process without a catalyst. The produced graphene films were of larger surface area, high quality and almost superhydrophobic with a stable contact angle of $135^{\circ}$. Even though the extract of tea tree comprised multicomponent, XPS surface analysis revealed no other element in the graphene, ascertaining the quality of the produced films. These graphene films are appropriate for electronic components such as memristors by sandwiching the semiconductor pentacene between graphene and aluminium films. This indicates the possible application of tea tree-derived graphene in resistive random access memory and non-volatile memory devices. As the precursor is a tea tree extract, a sustainable source, this method is environmentally friendly. However, the species from which the tea tree extract is obtained is only found in Australia and New Zealand, and hence further research is required to establish the applicability of this method in other countries with their native tea tree species.

A recent study used Colocasia esculenta (taro) and Nelumbo nucifera (Indian lotus) extracts to synthesize graphene nanocomposites using oxidative treatment by a modified Hummers' method [135]. The graphene synthesized from these extracts was reported to show inherent antimicrobial applications, which overcome the environmental issues that arise when using disinfectants such as hydrogen peroxide. The as-synthesized graphene nanocomposite was proved to be better or comparable to the common antibacterial drug clarithromycin in terms of biocidal activity. However, non-homogeneous particle size distribution was reported due to the accumulation of oxygenated groups in the graphene fold during surface passivation. The commercialization potential of this product is large, as the biocidal properties of the graphene nanocomposites are non-photocatalytic and hence function as an antibacterial agent under natural illumination unlike graphene derived from conventional sources [136].

The alfalfa plant (Medicago sativa L.) is promising for graphene synthesis as it contains several vascular bundles that can be used to prepare carbon nanostructures, and it is widely available globally. The alfalfa extracted is subjected to the oxidative action of nitric acid to form graphene sheets at $120^{\circ} \mathrm{C}$ [137]. Although the graphene sheets produced were agglomerated, the intrinsic wrinkled structure of graphene sheets could be observed. The nitric acid also works to remove some of the metal contained within plant cells, ensuring graphene of high purity. That being said, more work has to be done to improve the quality of the graphene sheets and prevent agglomeration; however, the low cost of the production process and the precursor make research in this area attractive. Graphene quantum dots, renowned for their optical applications, can be made from several plant extracts including neem, fenugreek, and nutmeg. Neem is favourable for its high hydrocarbon content paired with a low oxygen content while fenugreek is highly carbonaceous and contains several nitrogen groups. Quantum dots can be produced from these extracts using a one-pot hydrothermal method yielding highly thermal and $\mathrm{pH}$-stable quantum dots [138]. Extract from nutmeg seeds can be used as a precursor to graphene quantum dots through hydrothermal synthesis using hydrazine hydrate [139]. These quantum dots show good antimicrobial activity against several bacteria species including Salmonella sp. and Escherichia coli. As these antimicrobial properties are lent by the nutmeg seed extract, these graphene quantum dots have potential applications in the fields of drug delivery, bioimaging, and optical sensing.

The interest in plant-extract-based synthesis is clear, as no harsh chemicals, as well as process conditions, are involved. Obtaining usable, concentrated, and purified plant extracts is an energy-intensive task and the associated costs need to be considered along with the demand for specialized graphene. The applicability of these methods to similar plant extracts needs to be investigated to establish plant-based graphene, as most research is performed on highly localized plant species instead of common plants or invasive species. Furthermore, the applicability of this graphene in different areas from medical to electronic must be studied to make energy-intensive extraction for the synthesis of graphene.

\subsubsection{Natural oils}

Extensive studies of the ultra-structures of plant materials, such as oils, confirmed that these materials contain basic hydrocarbon compounds with skeletal structures [140], making them viable for the synthesis of graphene. Most plant materials, including seeds and oils, yield different forms of carbon when pyrolyzed, which explains the frequent featuring of pyrolysis in the synthesis methodology. Furthermore, these oils are much cheaper than hydrocarbons derived from fossil fuels besides being more environmentally friendly. Many of these oils could be used in their waste form after cooking, rather than in their original state, further reducing the carbon footprint when used in the synthesis of graphene. 
Kumar et al. [141] successfully synthesized graphene nanosheets via the spray pyrolysis of sesame oil assisted by ferrocene as a catalyst. The graphene nanosheets produced in this study were of six to eight layers thick, and no other functional groups were found on the surface of these graphene nanosheets. Further investigations found that these sesame-oil-derived graphene nanosheets were free of carbonaceous impurities. Therefore, no post-treatment on sesame-oil-derived graphene nanosheet was necessary. The need for temperature control is highlighted by the fluctuations in the stark temperature in the outer regions of the CVD furnace, which could not provide sufficient heat for graphitization, resulting in wrinkled aggregates forming disordered structures of graphene nanosheets [142].

Refined palm oil can be used to produce single-layer graphene on a copper substrate through spray injectorassisted CVD [143]. This method can achieve 97\% coverage with good crystallinity, but it is unable to control the amount of defects. The process will have to be modified to feature a rapid cooling mechanism to suppress graphene deposition after the single-layer has formed [144]. Waste cooking palm oil can be used to synthesize graphene utilizing the double thermal CVD method by incorporating a pretreatment furnace followed by a precursor furnace containing the nickel substrate [145]. This study highlighted the need to control the supply of precursor when the precursor supply was too low and the produced film exhibited porosity. Moreover, when the precursor supply was too high, defects were increased as carbon atoms were arranged in pentagonal or heptagonal structures resulting in misoriented graphene. However, with control in the supply of palm oil, 12-layer graphene sheets with very few defects were obtained. The applicability of graphene produced in this way has not been investigated with waste palm oil and hence a novel approach to green graphene synthesis; thus, more studies need to be performed on the properties exhibited by this product.

Producing graphene through natural oils is an ambitious process as rigorous control of operating parameters, including temperature and precursor supply, is essential to prevent graphene defects. Monolayer graphene has yet to be synthesized using natural oils, and thus, applications for the produced FLG need to be identified before such green synthesis of graphene is marketable. Furthermore, plant oils are seen as less environmentally damaging than other hydrocarbon precursors. Still, there are controversies associated with certain oils such as palm oil with doubts cast on the sustainability of palm oil plantations. If sustainably sourced palm oil is unavailable, the previously discussed synthesis methods work similarly when corn oil is substituted as a precursor [146]. To further combat waste generated from the palm oil industry, empty fruit bunches, which are usually discarded in the process, can be used to produce GO [147]. The specialized equipment for even heating is also required, as the defects within the stacked layers frequently resulted from uneven or overheating at boundary conditions. Considering the state of technology, more development and investigation in plant oils are required as the processes are still relatively novel and not optimized.

\subsubsection{Glucose}

Glucose is a plant-based monosaccharide, and hence it is a renewable and abundant carbon source. Various attempts at synthesizing graphene from glucose were reported, including carbonization and calcination of glucose and iron(III) chloride, $\mathrm{FeCl}_{3}$ mixture [148], and sugarblowing technique [149].

Zhang et al. [148] synthesized graphene sheets using carbonization and calcination of glucose and $\mathrm{FeCl}_{3}$ mixture. $\mathrm{FeCl}_{3}$ acted as both a template and a catalyst, facilitating the formation of graphene. The study highlighted the successful large-scale production of high-quality three-layer graphene sheets with electrical conductivity similar to those produced through CVD techniques [148]. However, this glucose-derived graphene suffered from yields as low as $40 \%$.

Wang et al. [149] successfully synthesized graphene from glucose using a novel sugar-blowing technique. Glucose and ammonium chloride were heated up to $1,350^{\circ} \mathrm{C}$ and subsequently polymerized in a tube furnace, leading to graphitization to form ultrathin graphitic membranes referred to strutted graphene. The struts in the structure prevent degradation of the graphitic membranes and agglomeration or restacking of graphene. The strutted graphene demonstrated high electrical conductivity, large surface area, and good mechanical strength and is, therefore, suitable for the applications of highpower-density supercapacitors. The porosity of the graphene also aids this application as the connected channels act as an electron pathway and maximize ionic conductivity and provide access to the surface. Thus, strutted graphene has been tested in the electrical double-layer capacitors, achieving maximum power densities of $893 \mathrm{~kW} \mathrm{~g}^{-1}$ at $100 \mathrm{~A} \mathrm{~g}^{-1}$ [149], which is comparable to an aluminium electrolytic capacitor and higher than several activated carbon and graphene compounds [150,151]. This suggests that applications in the area of fast 
charging of portable appliances are feasible due to high energy output in a limited time. However, the use of high temperatures may potentially be a drawback in terms of energy consumption.

Glucose should be seriously considered when the biocompatibility of graphene is a parameter that needs to be satisfied, such as in the use of graphene in medications and other therapeutics. Graphene sheets produced by other means are known to display cytotoxicity with reports of graphene sheets with sharp edges damaging cell membranes [152] and the trapping of cells within aggregated graphene sheets [153]. Several methods associated with other graphene synthesis techniques make use of strong reducing agents that are highly toxic and require further treatment to coat the material with polyethylene glycol, which further adds to the operating costs. Akhavan et al. [154] utilized glucose as a precursor to producing biocompatible $\mathrm{GO}$ sheets with significant water solubility by functionalizing the surface with gluconate ions in the presence of $\mathrm{Fe}$ catalyst. The graphene was able to destroy cancer cells in $12 \mathrm{~min}$, and while this rate is seen in therapies with hydrazine-reduced GO and multiwalled carbon nanotubes (MWCNTs), it displayed signs of cytotoxicity when used. From these studies, it can be surmised that the synthesis of graphene from glucose is not a simple process, featuring several processing steps and high temperatures. As a result, graphene produced in this strategy is significantly more expensive. However, a market exists for these products as they possess biocompatibility that very few graphene nanomaterials possess.

\subsubsection{Biomass}

Biomass such as rice husk [155], sugarcane bagasse [156], dead camphor leaves [157], waste papers [158], disposable paper cups [159], food, insects, waste [160], and others $[161,162]$ can be synthesized into graphene and its derivatives. Biomass is used to create value-added products which is an attractive prospect as it is a sustainable, non-toxic, environmentally friendly, cost-effective, and easily sourced precursor [163,164]. The suitability of biomass for graphene synthesis depends on chemical composition and property, mainly its carbon content and its texture in terms of mesoporosity to microporosity [165]. As a result, differing varieties of biomass have specific synthesis methods suitable for them (Figure 8). For example, the grainy, meso/macroporosity and high water content of pear have been exploited to generate graphene aerogels utilizing hydrothermal carbonization [166].

\section{Biomass-derived Graphene}

\section{Synthesis}

\section{Rice Husk}

Microwave Plasma Irradiation

\section{Sugarcane Bagasse}

Oxidation in a muffled atmosphere

\section{Camphor Leaves}

Thermal Pyrolysis

\section{Waste Paper}

Oxidation-degradation of Carbon Nanospheres

Figure 8: Biomass precursors and methods for graphene synthesis.

Wang et al. [155] synthesized graphene CNTs from rice husk by microwave plasma irradiation (MPI). The graphene CNTs are composed of 2- to 6-layer graphene sheets possessing several sharp edges, standing on the sidewalls of CNTs which were several tens of micrometres in length and 50-200 $\mathrm{nm}$ in diameter. These compounds show great promise for applications in electrochemical electrodes owing to their high surface area and specific capacitance. Since rice husk contains cellulose, hemicellulose, and lignin, which are the main components of most waste biomass, the success of this synthesis suggests that most waste biomass can potentially be used as feedstock to synthesize graphene CNTs through the MPI technique. This not only converts waste into higher value products, but it also assists in solving the environmental nuisance and disposal problems caused by huge quantities of waste biomass [155]. However, it has also been found that rice husk contains $17-20 \%$ of ash, in which over $90 \%$ is $\mathrm{SiO}_{2}$, with the rest being other metallic impurities [167]. Therefore, before employing rice husk as a starting material for the mass production of graphene, the issues on the purity of graphene must be considered. This issue has been addressed by Raghavan et al. [168], and a strong alkali, potassium hydroxide, was added to remove $\mathrm{SiO}_{2}$ impurities that assist in producing highpurity graphene containing stable and clean edges.

Somanathan et al. [156] synthesized GO from sugarcane bagasse (sugarcane oxidized under muffled atmosphere [SOMA]-GO) by directly oxidizing it under a muffled atmosphere. The sugarcane bagasse was crushed to obtain a powder which was then mixed with ferrocene and heated in a muffle furnace at $300^{\circ} \mathrm{C}$ for $10 \mathrm{~min}$ under atmospheric 
conditions. The powder was isolated from the fuel and combustion products, including gases and flying ash. At the end of the process, $\mathrm{GO}$ was collected at room temperature. This GO was characterized with various oxygen-containing functional groups (epoxy, hydroxyl, carboxylic, carbonyl, and others) that act as attachment sites for different biological molecules including proteins, DNA, and RNA and is also more hydrophilic than conventional graphene. Hence, it is more suitable for biotechnological and biomedical applications as the hydrophobicity of graphene causes several issues when introduced into the human system, which is mainly composed of water [169]. This method is relatively simple and environmentally friendly due to the repurposing of the agricultural waste and that it avoids toxic gas emissions during synthesis.

Shams et al. [157] synthesized FLG from dead camphor leaves by thermal pyrolysis without catalysts. The biomass was heated to $1,200^{\circ} \mathrm{C}$ with flowing nitrogen and then cooled down to room temperature. In this study, a few layers of graphene were successfully purified by $\pi-\pi$ interactions with D-tyrosine and centrifugation. This process appears to be relatively cheap, as the biomass source is of low cost and no catalyst is required. The need for temperatures as high as $1,200^{\circ} \mathrm{C}$ certainly contributes to higher operating costs. Furthermore, dead camphor leaves are not as widely available as the more common agricultural waste.

Adolfsson et al. [158] synthesized GO quantum dots (GOQDs) from waste papers that contain cellulose with amorphous carbon nanospheres (CNs) as the intermediates. CNs were first obtained as a by-product of microwave-assisted hydrothermal degradation of the waste papers. This was a result of the dehydration reaction of glucose, followed by a polymerization-precipitation reaction. CNs were then placed in an oxygen-enriched environment with nitric acid as the oxidizing agent where $\mathrm{CN}$ aggregates underwent disintegration by sonication. Then the main oxidation-degradation process occurred when the compounds were heated at $9^{\circ} \mathrm{C}$ to form nanosized sheets of GOQDs. The special chemical structure of GOQDs enabled them to absorb UV and visible light of lower wavelengths. GOQDs also possess fluorescent properties due to their optoelectronic state making them suitable for optoelectronic applications. These GOQDs can also be used in sensors, bio-imaging, drug-delivery systems, and solar cells [170]. The advantage of this method is that it uses renewable starting materials, i.e., waste papers.

Additionally, it upcycles the low-quality waste to value-added products. The synthesis condition is relatively mild with a maximum temperature of only $160^{\circ} \mathrm{C}$, but a pressure of around 10 bar is also required. Problems associated with the quality of waste paper-derived GOQDs, as food or other compounds on the paper, could lead to contamination. Therefore, proper pretreatment is necessary.

Zhao et al. [159] synthesized graphene sheets, iron/ graphene, and noble metals/graphene from disposable paper cups by graphitizing paper cup pulp in the presence of iron using an iron exchange process. During this process, few carbon atoms were incorporated into the iron phase to form a dense template of cementite, $\mathrm{Fe}_{3} \mathrm{C}$, layers. With a decrease in temperature, active carbon atoms in the $\mathrm{Fe}_{3} \mathrm{C}$ layers diffused out to form graphene on the surface of iron layers, and the excess carbon atoms precipitated on the top of the formed graphene layer. Due to this, the formed graphene sheets contained multilayers instead of monolayer graphene. The yield of graphene sheets per unit energy consumption was much higher than the conventional methods. The prepared graphene sheets were characterized with few identifiable defects but with high crystallinity. This process can be adapted for the generation of iron/graphene and noble metal/graphene sheets. Among the noble metals/graphene sheets synthesized as aforementioned, platinum/graphene sheets were reported to exhibit high catalytic activity for the oxygen reduction reaction in fuel cells [159].

Ruan et al. [160] synthesized monolayer graphene from a variety of sources including food, insects, and waste. This was carried out by growing graphene directly onto copper foil at $1,050^{\circ} \mathrm{C}$ with hydrogen and argon flow. The advantage of this method is that low-value carboncontaining materials can be used without pre-purification to produce high-quality monolayer graphene. Even though these carbon sources contain several other elements such as iron, sulphur, and phosphorus, these elements are not incorporated into graphene in significant amounts to disrupt its pristine structure. No pretreatment or cleaning of the solid materials is required before the synthesis of graphene, making the process even more efficient. The carbon precursors are inexpensive and fairly common. However, the feasibility of bringing this synthesis method to large-scale production is still unclear.

The ability to synthesize graphene from biomass is an attractive concept as a significantly high-value product could be obtained from waste materials. However, there are still challenges to be overcome before the realization of biomass-derived graphene. Large variations in the quality of graphene are found in the biomass sources, and synthesis of pristine graphene has not yet been 
achieved. Some of these processes require high operating temperatures (often exceeding $1,200^{\circ} \mathrm{C}$ ), which may negate the environmentally friendly aspect of utilizing biomass. The most significant biomass source and the process must be identified, and a collection system for accumulating this biomass must be implemented to ensure supply before the production of sustainable graphene.

\subsection{Chemical precursors}

Chemical precursors are distinct compounds that are chemically synthesized, rather than biologically or physically derived. These include common laboratory chemicals such as ethanol $\mathrm{CO}_{2}$ and $\mathrm{SiC}$. Synthetic polymers are also included, given their potential as a graphene precursor with several polymers are capable of making graphene of satisfactory quality.

\subsubsection{Synthetic polymers}

Synthetic polymers such as PMMA [171,172], polyimide $[173,174]$, and waste plastics $[175,176]$ were successfully converted into graphene. Sun et al. [171] synthesized graphene from PMMA by spin casting the polymer on a metal catalyst substrate, usually a film of nickel or copper deposited on a silicon dioxide/silicon $\left(\mathrm{SiO}_{2} / \mathrm{Si}\right)$ wafer. The synthesis temperature was kept at $800^{\circ} \mathrm{C}$ for $10 \mathrm{~min}$ under low-pressure conditions (between 8 and 15 Torr) with a constant flow of hydrogen and argon gases after which graphene was formed from the liquid-phase PMMA on the substrate. Hydrogen acts as both the reducing reagent and carrier gas to remove carbon atoms that were extruded from the decomposing PMMA during the growth phase. A slower hydrogen flow contains more carbon sources within the system for the growth of multilayer graphene, whereby the formation of higher order layers occurs through direct graphitization atop the first layer. This process allows good product control, as the thickness of PMMA-derived graphene can be varied by manipulating the flow rates of hydrogen and argon gases. This study also claimed to produce high-quality monolayer PMMA-derived graphene at the synthesis temperature lower than the CVD growth temperature on copper. Thus, this technique is more suitable for the semiconductor industry, where lower processing temperatures are favourable since temperatures as high as $1,000^{\circ} \mathrm{C}$ adversely affect the fabrication of multilayer stacks of heterogeneous materials in semiconductors. As PMMAderived graphene does not grow on $\mathrm{Si}$ or $\mathrm{SiO}_{2}$ surfaces, post-lithographic treatment is not required.

A $\mathrm{CO}_{2}$ infrared laser to produce laser-induced graphene (LIG) from polyimide films was attempted by Li et al. [177]. The resultant LIG was reported to exhibit high electrical conductivity and possessed defect-rich boundaries that enhanced the capacitance. The assynthesized LIG could be readily patterned to interdigitated electrodes for in-plane micro-supercapacitors. These properties endowed LIG to be used as energystorage devices. As polyimide is a common polymer, this process has the potential for large-scale applications. The application of a laser with a microsecond pulse indicates that this technique presents a high level of control and can respond in short reaction times. However, significant shrinkage of the polyimide films associated with the pyrolysis stage with linear shrinkage rates of up to $20-40 \%$ was noted, resulting in graphene with moderate to severe shape distortions [173].

As waste plastic is rich in polyethylene and PS [176], it can be used as a precursor to synthesize graphene on copper foil at atmospheric pressure via a CVD method $[175,176]$. This process is environmentally friendly, as waste can be transformed into value-added products. Besides, this process can be performed at a low pyrolysis rate to reduce the injection rate of carbon radicals to the atmosphere [176]. However, this method is unsuitable for the semiconductor industry, due to its high annealing temperature of $1,000^{\circ} \mathrm{C}[175,176]$.

Polymers are much more affordable starting materials and easily deposited onto any substrate, making inkjet printing [178] of graphene (a digital lithography technique) a possibility that significantly aids the adoption of patterned graphene in electronics. Polymer-derived graphene poses the advantage of being synthesized under less extreme temperatures and pressures; conditions favoured by the semiconductor industry. Furthermore, common polymers, such as PMMA, are safe to handle, unlike the strong acids associated with the Hummers' method and the explosive carbon sources used in few CVD processes [179]. Agglomeration of graphene layers is still a problem associated with a polymeric synthesis that has not been fully addressed in the current literature. Efforts have been made to use surfactants [180] as stabilizers or disperse the graphene layers in water [181] with varying degrees of success. A polymeric ionic liquid (PIL) shows promise in its ability to stabilize isolated graphene sheets, as the strong $\pi-\pi$ bonds and van der Waals forces of attraction between graphene layers are shielded by the PIL [182]. The exact mechanism of this 
interaction is still not understood, with the requirement of more investigations into this area before the production of polymer-derived graphene can be scaled up.

\subsubsection{SiC}

$\mathrm{SiC}$ is another popular choice as a substrate for the growth of graphene. Still it can also be used for graphene synthesis via the epitaxial growth method as previously described by Kaushik et al. [183]. The $\mathrm{SiO}_{2}$ carbidederived graphene is suitable for gas sensing, biosensing, magneto-sensing, electrochemical, and spintronic applications. The advantage of this approach is that large areas of monolayer graphene and FLG can be grown directly on the insulating $\mathrm{SiC}$ substrate. Thus, transfer to another insulating substrate is unnecessary, and it can easily be transferred onto arbitrary substrates if required. As transferring is not required, epitaxial graphene does not suffer from the same contamination levels experienced by graphene synthesized via CVD technique from processing the pollutants [183]. It does require highly specialized equipment and the $\mathrm{SiC}$ substrate is expensive, making the whole process economically unfeasible. Furthermore, when epitaxial growth occurs on SiC-based semiconductors, the step bunching of the SiC surface greatly affects the structure of the graphene layer [184].

Several other synthesis methods utilizing SiC are available but are not widely studied. The thermal decomposition of $\mathrm{SiC}$ [185] can be used to produce wafer-like graphene for technological applications; and as insulating $\mathrm{SiC}$ substrates can be used, transfer to another insulator is not required. However, the film produced lacks uniformity and continuity as thermal decomposition of $\mathrm{SiC}$ is also not a self-limiting process, and hence graphene regions with different thicknesses are often generated [5]. There is some evidence to suggest the step bunching encountered through epitaxial growth can be overcome by utilizing the pyrolysis of $6 \mathrm{H}-\mathrm{SiC}$ crystals [186]. According to the desired specifications, the temperature, argon flow, and growth pressure can be controlled to produce mono-graphene. If this proves commercially applicable, $\mathrm{SiC}$ will be a more viable option in its ability to produce a high-quality graphene film.

\subsubsection{Solid $\mathrm{CO}_{2}$}

$\mathrm{CO}_{2}$ is well known as a major greenhouse gas [187]; thus, efforts are continuously attempted to reduce its emissions by converting it to useful products such as graphene.
While $\mathrm{CO}_{2}$ is a good carbon source for graphene synthesis, its solid form, dry ice, is more commonly used. This is because the process relies on the supercritical state of $\mathrm{CO}_{2}$ at high temperatures.

Chen et al. [188] synthesized FLG sheets from dry ice by the reduction of solid $\mathrm{CO}_{2}$ with calcium hydride under shock wave loading. Shock wave action can induce the redox reaction between $\mathrm{CO}_{2}$ and calcium hydride, forming FLG nanosheets within a brief period. By adding ammonium nitrate as a doping agent, $\mathrm{N}$-doped graphene was then formed in one step. This graphene was observed to act as a metal-free electrode with efficient electrocatalytic activity towards the oxygen reduction reaction in alkaline solutions.

Several studies based on $\mathrm{CO}_{2}$-derived graphene utilized high pressure or supercritical $\mathrm{CO}_{2}$, which requires extra energy expenditure to process the gas from its atmospheric state. Recent research focused on converting gaseous $\mathrm{CO}_{2}$ to graphene at a relatively low temperature of $680^{\circ} \mathrm{C}$ by utilizing a magnesium reducing agent and nickel nanoparticles [189]. The graphene produced was of high crystallinity and low electrical resistivity, suggesting potential for using this material in $\mathrm{Li}-\mathrm{S}$ batteries after sulphur impregnation. However, graphene yield was low at $11.2 \%$ due to concurrent reactions of magnesium and $\mathrm{CO}_{2}$. Therefore, more studies are required to ascertain methods of repressing these side reactions to obtain a higher yield. A similar study featuring $\mathrm{CO}_{2}$ reduction, however, in this instance, with lithium as a reducing agent, found graphene produced in this way had fewer defects than chemically reduced graphene [190]. As graphite is a mineral, residual impurities are very common, especially in the graphite of industrial grade, which can affect the electrochemistry of the resultant graphene. Graphene synthesized using $\mathrm{CO}_{2}$ contains very-low-residual metallic impurities compared to levels found in chemically reduced graphene. This is beneficial as metallic impurities significantly affect the electrochemical and toxicological properties of graphene, particularly Fe and Ni impurities [191].

As $\mathrm{CO}_{2}$ is a cheap, readily available greenhouse gas, the incentive to make value-added products using it as a starting material is large. Unfortunately, the gas cannot be immediately used in its gaseous form, which usually requires conversion to dry ice or supercritical $\mathrm{CO}_{2}$ which are extremely energy intensive. Recent studies have suggested that it is possible to synthesize $\mathrm{CO}_{2}$-derived graphene under atmospheric conditions, where the yields remain low, necessitating further research into reducing agents and controlling side reactions before its commercialization. 


\subsubsection{Ethanol and other alcohols}

Alcohols such as methanol, ethanol, and 1-propanol are comparatively cheaper, easier to use, and less flammable than high-purity methane and thus advantageous as liquid precursors for graphene growth [192]. Ethanol [192-195] and other alcohols such as methanol [192], 1-propanol [192], and 2-phenylethanol [195] were used to synthesize monolayer, bilayer, and trilayer graphenes using the atmospheric pressure CVD method with a single-gas injection line supplying hydrogen and argon. Chen et al. [193] reported that a decrease in ethanol pressure during CVD caused the transition from the production of a self-limited single-layer to multilayer graphene, where a narrow window was preferred for the formation of large-domain bilayer graphene. This suggests that ethanol played two roles in graphene growth: one was to act as the carbon precursor, and the other was to determine the number of layers in the resultant graphene [193]. The process temperature greatly affected the crystal quality of the graphene with an increase in temperature from $650^{\circ} \mathrm{C}$ to $850^{\circ} \mathrm{C}$, increasing the graphene crystal domain size of up to $168 \mathrm{~nm}$ [196]. High hydrogen flow rates, above $100 \mathrm{sccm}$ [197], were exploited to produce multilayer graphene as hydrogen could impede etching of water produced through the decomposition of ethanol. This method is rapid, easy, and scalable to industrial production. The use of ethanol might also represent an advantage for high-end reactors simplified by eliminating hazardous gas lines and pressurized cylinders [194].

Alcohols can also be used to produce single-crystal graphene grains that are used in microelectronics. To produce these grains, nucleation density has to be reduced to prevent grains from forming a film. This can be achieved by utilizing oxygen-treated $\mathrm{Cu}$ foils as the substrate, producing graphene grains of up to $500 \mu \mathrm{m}$ [198]. Furthermore, this method does not require copper structuring, such as the use of folded enclosures or sealing. However, this method requires high temperatures, exceeding $1,000^{\circ} \mathrm{C}$ [199], to produce high-quality crystals.

As ethanol decomposes at relatively lower temperatures than precursors such as methane, graphene can grow at a lower temperature with less energy demands. Alcohols, used for syntheses, such as ethanol and propanol, are liquids under atmospheric conditions, allowing them to be supplied with an inert carrier at low pressure, thus negating the need for the highly flammable, pressurized gas cylinders required in the CVD system. The quality of alcohol-derived graphene films was comparable to that of methane-derived graphene, and the process time to grow a complete film was greatly reduced. Growth temperatures were higher than that of the methane process as carbon atoms have lower mobility on the metal substrate surface when using ethanol. Thus, while the graphene produced through ethanol was proved to be fairly favourable over methane synthesis, greater care must be taken for temperature and pressure control.

\subsection{Fossil fuel precursors}

\subsubsection{Coal}

Coal is a fossil fuel found abundantly worldwide and is a key energy source for electricity generation in several countries. Coal is an inexpensive resource yet possesses a high carbon content [200]. Many studies are based on the thermal behaviours of coal in the energy field [201]. However, the role of coal as fossil fuel is discouraged, particularly after the Paris Agreement guidelines [202], as its direct combustion releases greenhouse gases and other polluting components if the exhaust is not treated first [203]. Thus, utilizing coal instead of producing value-added materials such as graphene is advantageous both environmentally and economically.

Vijapur et al. [204] synthesized graphene from subbituminous coal through pyrolysis. First, sub-bituminous coal was heated in the absence of oxygen. This pyrolysis allowed coal to decompose into hydrocarbon gases, which are indirect precursors thermally. Then the carbon compounds were adsorbed onto copper, producing a hybrid carbon film, which was then graphitized in the presence of hydrogen to form graphene domains which merged to form graphene films [204]. These graphene films could then be transferred to various substrates, demonstrating significant promise for applications in solar cells, light-emitting diodes, photodiodes, and biomedical implants. Furthermore, the as-synthesized graphene film was uniform and transparent, making it suitable for optical and magnetic storage applications. However, it must be acknowledged that there is a chance of contamination or physical damage during the transfer process. As this process involves pyrolysis, there are still some questions about its environmental impact, as several polluting gases are generated which are not captured in the carbon film.

Several other attempts were made to synthesize graphene from bituminous coal [55] and lignite coal [205]. Wang et al. [206] successfully synthesized graphene from 
bituminous coal by first electrolyzing the coal to obtain its by-product, coal char, and then a CVD process to form graphene film. Through this process, complete graphene films with large areas were produced. The resultant graphene films could be directly used as anodes for lithiumion batteries without the aid of polymer binders and conductive fillers as they were grown on copper substrates. Besides lithium-ion batteries, these graphene anodes have potential applications in thin-film batteries to power micro- or nanodevices, such as implantable medical micromachines and on-chip memory. However, due to the transfer process, wrinkles, back-folding and overlapping of edges were also formed, which may adversely affect the properties of graphene.

In another study, Powell et al. [205] synthesized humic acid (HA) powders, which were GO-like sheets, from lignite coal. The lignite coal was placed in a basic aqueous solution of $\mathrm{pH} 10$ to extract leonardite, which was then precipitated from the solution by acidification to produce HA powder. The particles derived from HA were useful for applications requiring individual GO particles such as polymer nanocomposites. However, in applications where electrical properties are of interest, especially over the larger areas in current display devices, graphene films produced from HA and reduced HA are not conductive enough to be of practical importance [205].

Due to the non-renewable nature of coal, there is precedent to make use of material no longer suitable for energy generation, unlike the pristine coal used in the studies as mentioned earlier. Considering this, coal tar pitch (CTP) is a viable option for graphene synthesis as it is a by-product of the coal tar distillation process. Liu et al. [207] used the in situ $\mathrm{KOH}$ activation technique and a zinc template to produce GO from CTP. The CTP-derived GO was highly functional, consisting of $21 \%$ oxygen functional groups, rendering it useful in applications such as adsorption. Some contamination from the zinc template used was observed through an EDS study; however, the zinc content was less than 0.1 at $\%$ [207], which is unlikely to affect the performance in most applications.

While the position of coal as a non-renewable resource and emitter of pollutant gases in specific applications cannot be ignored, it can produce graphene of good quality in terms of uniformity with satisfactory yields. If pyrolysis is necessary to the process, extra processing steps should be incorporated to ensure minimal hydrocarbon compounds are lost, and the exhaust is treated before release. Furthermore, conventional graphene production methods such as Hummers' are timeconsuming [208] and run the risk of explosion, unlike synthesis through coal which has the added advantage of a relatively low-cost starting material.

\subsubsection{Methane and liquefied petroleum gas (LPG)}

Synthesis of graphene from methane can be carried out using the CVD method. Due to the strong $\mathrm{C}-\mathrm{H}$ bonds in methane molecules (440 kJ/mole), its thermal decomposition occurs at very high temperatures of above $1,200^{\circ} \mathrm{C}$. Different transition metal catalysts such as iron, cobalt, nickel, and copper were used to reduce the minimum temperature of methane's thermal decomposition. Among these transition metals, the decomposition rate of methane on copper is the lowest as copper cannot form a carbide with carbon, which results in the low solubility of carbon in copper [209]. On the other hand, iron, cobalt, and nickel have a higher-than-desirable capability to decompose hydrocarbons and generally form few layers of graphene [210]. However, nickel catalysts have their advantages such as easy and effective removal by a simple iron(III) chloride/hydrochloric acid treatment without compromising the quality of the graphene sheets. The purified graphene sheets are of high quality with excellent crystallinity, low electrical resistance, and high oxidation resistance. This method can be used for the large-scale production of high-quality graphene sheets, which may facilitate a wide range of applications of graphene, including in composites, energy storage, transparent conductive films, and field emitters [211].

The CVD process can also be adapted for graphene synthesis using LPG which is a low-cost precursor mainly consisting of butane and propane. The process parameters of growth time, temperature, gas amount, and flow rate need to be carefully controlled to produce high-quality graphene [211]. Hydrogen gas is also introduced to the system with the precursor, which slows down the growth rate compared to precursor system. Still it leads to a higher quality product due to the alternate growth and etching processes. Synthesis using LPG is 10 times faster than using methane; however, due to the trace amounts of sulphur that are conventionally found in LPG, several impurities are observed in the graphene as sulphur can form covalent bonds along with the defects on the graphene surface.

While LPG is a clean-burning gas mainly derived from fossil fuels, presenting it as an environmentally friendly precursor may be debated. On the other hand, methane can be produced from organic waste and thus is not only reliant on the extraction from bedrock. Several advances have been made in biogas technology and even 
plans are in place to harness methane from ruminants, ensuring methane supply for mass synthesis of graphene. However, the efficiency of synthesis using LPG over methane cannot be denied. For both these methods, extremely high temperatures are needed to decompose gas to yield its carbon which makes the process very energy intensive. Once methods are developed to stem the expression of sulphur impurities in the graphene produced, LPG will become a more viable option. Still, currently, methane is most suited for the commercial manufacturing of graphene.

\subsection{Advanced starting material}

\subsubsection{CNTs}

Graphene can also be synthesized by unzipping CNTs [212]. CNTs are cylindrically rolled graphene sheets with high aspect ratios as their diameters are usually only a few nanometres wide [213]. The CNTs frequently used for graphene synthesis are multiwalled CNTs (MWCNTs), which are comprised of several concentric graphene cylinders and thus can have diameters of up to $150 \mathrm{~nm}$ [214]. Various methods are available to unzip CNTs, such as the microwave CVD [215], cryo-milling [216], and chemical treatment followed by thermal reduction [217], among others.

Silva et al. [215] synthesized graphene sheets from unzipped MWCNTs using the microwave CVD method, which requires a microwave source of $800 \mathrm{~W}$ to heat the pristine MWCNTs. The procedure to unzip MWCNTs is similar to the one to synthesize MWCNTs, with only the initial hydrogen flow, the proportion of hydrogen/ methane gas mixture, and the pressure in the reactor being slightly higher to produce the unzipped MWCNTs. This is necessary as the reactivity of the gas phase with the MWCNTs surface increases with an increase in the proportion of hydrogen, enabling the breaking of $\mathrm{sp}^{2}$ bonds in MWCNTs. Thus, defects on the MWCNTs increased, leading to the unzipping of MWCNT and the opening of graphene sheets. This process allowed the unzipping of MWCNTs with no stacking of the graphene sheets, ensuring the production of single-layer graphene sheets. Notably, the unzipped MWCNTs exhibited high double-layer charging due to its good electron transfer rate. Hence, they are more suitable to be used as carbonbased supercapacitor devices.

Tiwary et al. [216] synthesized graphene particles by milling MWCNTs at a cryogenic temperature of $150 \mathrm{~K}$. The high strain exerted by the ball mill causes the MWCNTs to deform and become powder. The powder was then dispersed into a mixture of methyl alcohol and water. Subsequently, the solution was subjected to $15 \mathrm{~min}$ of ultrasonication, which resulted in a homogeneous dispersion of CNTs/graphene. The low temperature is essential to avoid local melting, cold welding, and bridging of the MWCNTs due to the high strain rate. The advantage of this process is that no chemical treatment is necessary, and, thus, the environmental impact is reduced.

Kosynkin et al. [217] synthesized oxidized graphene nanoribbons (GNRs) from MWCNTs through chemical treatment, which is known as the longitudinal unzipping process. This material was then thermally reduced in $\mathrm{H}_{2}$ to form usable GNRs, promising electrode materials for supercapacitors [218]. While the same technique can theoretically be applied to single-walled CNTs, the assynthesized GNRs were prone to entanglement, after which the separation of individual GNRs was a challenge. The oxygenic functional groups on the GNRs substantially reduced the conductivity of the product. However, this can be improved through chemical reduction using hydrazine or annealing with hydrogen.

Several studies concluded that graphene synthesis from CNTs is possible and confers properties to graphene such as good electron transfer rates. However, the unzipping process greatly relies on the deformation and destruction of the CNTs, which can cause significant damage to the structure of the material that is later transferred to the graphene product. Studies performed by Xiao et al. [219] demonstrated that continuous etching in the unzipping process could contribute to fragmentation in graphene, resulting in aggregation and a significant drop in the surface area. As most experiments are performed with commercially purified MWCNTs, industrial-grade MWCNTs [220] should be investigated as a low-cost alternative to make the manufacturing of graphene through this method economically viable. There are issues related to the raw material itself as very little is known about the effect of CNTs on human health and the environmental implications associated with their use. The ultrafine nature of this material means inhalation is the key exposure pathway [221] and may produce asbestos-like effects on entry to the respiratory tract.

\subsubsection{Carbon ions}

A novel approach to graphene synthesis was attempted by Kim et al. [222] which featured the successful synthesis of multilayer graphene from carbon ions. This method 
featured a nickel film as a catalyst, which was selected for its high carbon solubility, and a silicon substrate at a satisfactory temperature of $500^{\circ} \mathrm{C}$. A rapid thermal processing technique was used to increase the average nickel grain size, after which carbon could be implanted. Temperatures were kept low to minimize implantationinduced damage and reduce the activation annealing temperature. It was indicated that the ion implantation method could be used to synthesize graphene precisely with a certain layer of thickness by controlling the dose of carbon ion implantation. This graphene can be used in Si microelectronics, unlike the graphene produced through CVD or transfer processes. While highly functional for use in several applications, this method has not yet been developed to industrial-scale production due to the sophistication level.

\subsection{Potential and applicability of proposed precursors}

Figure 9 summarized the potential graphene precursors and the applicability of proposed precursors. The conventional precursors have several established synthesis methods that have already been widely investigated. Graphite-derived graphene through continuous mechanical cleavage and electrochemical exfoliation methods are most suitable for large-scale production and can produce monolayer graphene or FLG. However, graphene production needs to veer away from the extensive use of graphite as a starting material, given the limited stores of the material and its high cost. GO can be used as a precursor through the electrochemical reduction method even though the chemical reduction is the current convention. This negates the need for toxic chemical reducing agents such as hydrazine. However, GO supplies face the same downfalls as that associated with graphite produced from pure graphite.

When using plant- or animal-derived precursors, the CVD technique is the most appropriate as it is the best to maintain the uniformity of the sheets or particles produced and can maintain the electrical properties of graphene. The exception would be using waste biomass, where pyrolysis is the preferred synthesis method to release and isolate the carbon particles to form graphene. Some plant precursors are particular to certain geographic regions, so it is unclear whether the synthesis will be as effective with different plant species. Furthermore, it is recommended that these precursors be sourced from waste, so that the use of food sources can be otherwise consumed.
Potential Graphene Precursors \& Synthesis

Methods

\section{Conventional Precursors - Graphite \& GO}

- CVD method

Plant- \& Animal-Derived Precursors

- CVD most appropriate

- Pyrolysis preferred for waste biomass

\section{Chemical Precursors}

- CVD with PMMA for polymer-derived graphene

- Epitaxial growth for silicon carbide precursor

- Chemical reduction for carbon dioxide precursor

- Alcohol-derived graphene best produced through CVD

\section{Fossil Fuels}

- CVD most suitable

- CVD utilizing $\mathrm{KOH}$ activation preferred for coal tar pitch

Advanced Starting Materials

- Cryogenic synthesis for CNT precursor

- Carbon ion-derived precursor currently produced through thermal processing

Figure 9: Summary of potential graphene precursors and synthesis methods.

As the range of chemical precursors available for synthesis is wider, a general synthesis method most appropriate for the entire group cannot be stated. The most established route for synthetic polymer-derived graphene is through CVD utilizing PMMA. This method can be used in the semiconductor industry and does not require energy-intensive equipment such as employed in the novel laser synthesis techniques. For $\mathrm{SiC}$ as a precursor, epitaxial growth has been the most widely studied owing to its ability to produce large areas of monolayer graphene and FLG. It thus will be the synthesis method of choice from an industrial standpoint. While novel techniques for synthesis with $\mathrm{CO}_{2}$ exist, their yields are too low for consideration, and thus chemical reduction remains the most appropriate method. Alcohol-derived graphene can be satisfactorily produced through CVD; however, rigorous control is needed. When utilizing CNTs, cryogenic synthesis is preferred to avoid complex unzipping of tubes. The synthesis of graphene from carbon ions through thermal processing is still a novel concept and is studied in greater detail before it can be considered for mass production.

Due to their contribution to global warming, fossil fuel is being discouraged in several industries. However, coal is an attractive option for graphene synthesis as it produces high-quality graphene which can be used in 
several applications from electronics to storage devices and biomedical implants. As a result, it is recommended that CTP is used to produce graphene through the CVD technique utilizing $\mathrm{KOH}$ activation. Methane and LPG are cleaner-burning fossil fuels and so may be preferable to coal. These precursors are the most suited for the CVD technique; however, success greatly depends on the substrate used, with nickel being the most appropriate.

\section{Conclusion}

Graphene's unique properties make it suitable for a wide range of applications, and thus, it is a valuable material with high demand from several industries. To address this, intense studies have been conducted to find the best method of synthesizing high-quality graphene and on a large-scale basis using the most appropriate precursors. In general, graphene can be synthesized from either the top-down or bottom-up approaches. The top-down approach is the synthesis of graphene by delaminating large carbonaceous materials into single-layer graphene or FLG, whereas the bottom-up approach is the assembly of graphene from smaller carbon units. Common topdown approaches for the synthesis of graphene are exfoliation and reduction, while the typical bottom-up approaches for the synthesis of graphene are CVD, epitaxial growth, and pyrolysis.

Several different materials can be used as precursors to synthesize graphene, with varying degrees of success. Most conventional precursors are in solid forms; however, liquid and gas precursors have also been proved effective. Although renewable resources as precursors are ideal, these materials should be thoroughly evaluated and considered to ensure that the environmental impacts associated with all the stages of the renewable-resourcederived graphene are low. In some cases, extreme operating conditions such as high temperatures or pressures make graphene synthesis energy intensive and economically unfeasible. Furthermore, very few methods can synthesize pristine graphene with defects often found along the boundaries of the structures synthesized with great detriment to the favourable properties of graphene. To conclude, the selection of appropriate precursors is fundamental to the quality of graphene and the success of graphene products on a market scale. The selection should account for processing conditions, precursor availability, and quality of graphene produced. As graphene is beginning to be explored in every widening array of applications, it is important that synthesis convention is not confined to one method or one precursor, rather allowing varying types of graphene to produce through a myriad of processes to find its use in many different industries.

Acknowledgements: The authors gratefully express gratitude to all parties who have contributed towards the success of this project, both financially and technically, especially the S \& T Innovation 2025 Major Special Programme [grant number 2018B10022] and the Ningbo Natural Science Foundation Programme [grant number 2018A610069] funded by the Ningbo Science and Technology Bureau, China as well as the UNNC FoSE Faculty Inspiration Grant, China. The Zhejiang Provincial Department of Science and Technology is also acknowledged for this research under its Provincial Key Laboratory Programme (2020E10018).

Conflict of interest: The authors declare no conflict of interest regarding the publication of this paper.

\section{References}

[1] Inagaki M, Kang F, Toyodo M, Konno H. Graphene: Synthesis and preparation. In: Advanced materials science and engineering of carbon. Oxford, UK: Butterworth-Heinemann; 2014. p. 41-65.

[2] Dreyer DR, Sungjin P, Bielawski CW. The chemistry of graphene oxide. Chem Soc Rev. 2010;39(1):228.

[3] Novoselov KS, Geim AK, Morozov SV, Jiang D, Zhang Y, Dubonos SV, et al. Electric field effect in atomically thin carbon films. Science. 2004;306(5696):666-9.

[4] Van Wetering K, Pinczuk A. The 2010 Nobel Prize in Physics. Solid State Commun. 2010;45(150):2207.

[5] Whitener Jr KE, Sheehan PE. Graphene synthesis. Diam Relat Mater. 2014;46:25-34.

[6] Zhu Y, Ji H, Cheng HM, Ruoff RS. Mass production and industrial applications of graphene materials. Natl Sci Rev. 2018;5(1):90-101.

[7] Lin L, Peng H, Liu Z. Synthesis challenges for graphene industry. Nat Mater. 2019;18(6):520-4.

[8] Kong W, Kum H, Bae SH, Shim J, Kim H, Kong L, et al. Path towards graphene commercialization from lab to market. Nat Nanotechnol. 2019;14(10):927-38.

[9] Banhart F, Kotakoski J, Krasheninnikov AV. Structural defects in graphene. ACS Nano. 2011;5(1):26-41.

[10] Kauling AP, Seefeldt AT, Pisoni DP, Pradeep RC, Bentini R, Oliveira RV, et al. The worldwide graphene flake production. Adv Mater. 2018;30(44):1803784.

[11] Duong DL, Han GH, Lee SM, Gunes F, Kim ES, Kim ST, et al. Probing graphene grain boundaries with optical microscopy. Nature. 2012;490(7419):235-9.

[12] Wolf EL. Applications of graphene: An overview. 1st ed. Cham: Springer International Publishing; 2014. p. 1-18 
[13] Lim JY, Mubarak NM, Abdullah EC, Nizamuddin S, Khalid M. Recent trends in the synthesis of graphene and graphene oxide based nanomaterials for removal of heavy metals: A review. J Ind Eng Chem. 2018;66:29-44.

[14] Vignesh S, Surendran R, Sekar T, Rajeswari B. Ballistic impact analysis of graphene nanosheets reinforced kevlar29. Materials Today: Proceedings [Internet]; 2020 [cited 2020 Nov 06]. Available from https://www.sciencedirect.com/science/article/pii/S2214785320315984 Subscription required

[15] Liu Y, Jia M, Song C, Lu S, Wang H, Zhang G, et al. Enhancing ultra-early strength of sulphoaluminate cement-based materials by incorporating graphene oxide. Nanotechnol Rev. 2020;9(1):17-27.

[16] Wang J, Xu Y, Wu X, Zhang P, Hu S. Advances of graphene-and graphene oxide-modified cementitious materials. Nanotechnol Rev. 2020;9(1):465-77.

[17] Guo K, Miao H, Liu L, Zhou J, Liu M. Effect of graphene oxide on chloride penetration resistance of recycled concrete. Nanotechnol Rev. 2019;8(1):681-9.

[18] Lin M, Wang ZL, Yang PW, Li P. Micro-structure and rheological properties of graphene oxide rubber asphalt. Nanotechnol Rev. 2019;8(1):227-35.

[19] Wang J, Jin X, Li C, Wang W, Wu H, Guo S. Graphene and graphene derivatives toughening polymers: Toward high toughness and strength. Chem Eng J. 2019;370:831-54.

[20] Behdinan K, Moradi-Dastjerdi R, Safaei B, Qin Z, Chu F, Hui D. Graphene and CNT impact on heat transfer response of nanocomposite cylinders. Nanotechnol Rev. 2020;9(1):41-52.

[21] Naghib SM, Zare Y, Rhee KY. A facile and simple approach to synthesis and characterization of methacrylated graphene oxide nanostructured polyaniline nanocomposites. Nanotechnol Rev. 2020;9(1):53-60.

[22] Liu Y, Jiang X, Shi J, Luo Y, Tang Y, Wu Q, et al. Research on the interface properties and strengthening-toughening mechanism of nanocarbon-toughened ceramic matrix composites. Nanotechnol Rev. 2020;9(1):190-208.

[23] Glukharev AG, Konakov VG. Synthesis and properties of zirconia-graphene composite ceramics: A brief review. Rev Adv Mater Sci. 2018;56(1):124-38.

[24] Konakov VG, Kurapova OY, Solovyeva EN, Lomakin IV, Archakov IY. Synthesis, structure and mechanical properties of bulk "copper-graphene" composites. Rev Adv Mater Sci. 2018;57(2):151-7.

[25] Khatkar SK, Suri NM, Kant S. A review on mechanical and tribological properties of graphite reinforced self lubricating hybrid metal matrix composites. Rev Adv Mater Sci. 2018;56(1):1-20.

[26] Zhang S, Wang H, Liu J, Bao C. Measuring the specific surface area of monolayer graphene oxide in water. Mater Lett. 2020;261:127098.

[27] Wong SI, Sunarso J, Wong BT, Lin H, Yu A, Jia B. Towards enhanced energy density of graphene-based supercapacitors: Current status, approaches, and future directions. J Power Sources. 2018;396:182-206.

[28] Wang Y, Pan C, Chu W, Vipin AK, Sun L. Environmental remediation applications of carbon nanotubes and graphene oxide: Adsorption and catalysis. Nanomaterials. 2019;9(3):439.
[29] Sherlala AI, Raman AA, Bello MM, Buthiyappan A. Adsorption of arsenic using chitosan magnetic graphene oxide nanocomposite. J Environ Manag. 2019;246:547-56.

[30] Lingamdinne LP, Koduru JR, Karri RR. A comprehensive review of applications of magnetic graphene oxide based nanocomposites for sustainable water purification. J Environ Manag. 2019;231:622-34.

[31] Yan Y, Shin WI, Chen H, Lee SM, Manickam S, Hanson S, et al. A recent trend: application of graphene in catalysis. Carbon Lett. 2020;30:1-23.

[32] Hou P, Xing G, Tian L, Zhang G, Wang H, Yu C, et al. Hollow carbon spheres/graphene hybrid aerogels as high-performance adsorbents for organic pollution. Sep Purif Technol. 2019;213:524-32.

[33] Iqbal MZ, Rehman AU. Recent progress in graphene incorporated solar cell devices. Sol Energy. 2018;169:634-47.

[34] Mahmoudi T, Wang Y, Hahn YB. Graphene and its derivatives for solar cells application. Nano Energy. 2018;47:51-65.

[35] Kalita G, Matsushima M, Uchida H, Wakita K, Umeno M. Graphene constructed carbon thin films as transparent electrodes for solar cell applications. J Mater Chem. 2010;20(43):9713-7.

[36] Hernandez Y, Pang S, Feng X, Müllen K. Graphene and its synthesis. Polymer science: A comprehensive reference. 1st ed. Amsterdam: Elsevier Science; 2012. p. 415-38

[37] Verma S, Mohanty S, Nayak SK. Preparation of hydrophobic epoxy-polydimethylsiloxane-graphene oxide nanocomposite coatings for antifouling application. Soft Matter. 2020;16(5):1211-26.

[38] Ghany NA, Elsherif SA, Handal HT. Revolution of graphene for different applications: State-of-the-art. Surf Interfaces. 2017;9:93-106.

[39] Zhong Y, Zhen Z, Zhu H. Graphene: Fundamental research and potential applications. FlatChem. 2017;4:20-32.

[40] Fan Y, Xiang Y, Shen HS. Temperature-dependent negative Poisson's ratio of monolayer graphene: Prediction from molecular dynamics simulations. Nanotechnol Rev. 2019;8(1):415-21.

[41] Ahmad H, Fan M, Hui D. Graphene oxide incorporated functional materials: A review. Compos Part B Eng. 2018;145:270-80.

[42] Sun M, Li J. Graphene oxide membranes: Functional structures, preparation and environmental applications. Nano Today. 2018;20:121-37.

[43] Lee J, Kim J, Kim S, Min DH. Biosensors based on graphene oxide and its biomedical application. Adv Drug Deliv Rev. 2016;105:275-87.

[44] Kuang B, Song W, Ning M, Li J, Zhao Z, Guo D, et al. Chemical reduction dependent dielectric properties and dielectric loss mechanism of reduced graphene oxide. Carbon. 2018;127:209-17.

[45] Du S, Chen H, Hong R. Preparation and electromagnetic properties characterization of reduced graphene oxide/ strontium hexaferrite nanocomposites. Nanotechnol Rev. 2020;9(1):105-14.

[46] Ghouri ZK, Motlak M, Afaq S, Barakat NA, Abdala A. Template-free synthesis of Se-nanorods-rGO nanocomposite for application in supercapacitors. Nanotechnol Rev. 2019;8(1):661-70. 
[47] Afre RA, Sharma N, Sharon M, Sharon M. Transparent conducting oxide films for various applications: A review. Rev Adv Mater Sci. 2018;53(1):79-89.

[48] Lee HC, Liu WW, Chai SP, Mohamed AR, Lai CW, Khe CS, et al. Synthesis of single-layer graphene: A review of recent development. Procedia Chem. 2016;19:916-21.

[49] Zhang Q, Wu Z, Li N, Pu Y, Wang B, Zhang T, et al. Advanced review of graphene-based nanomaterials in drug delivery systems: Synthesis, modification, toxicity and application. Mater Sci Eng C. 2017;77:1363-75.

[50] Lee XJ, Hiew BY, Lai KC, Lee LY, Gan S, ThangalazhyGopakumar S, et al. Review on graphene and its derivatives: Synthesis methods and potential industrial implementation. J Taiwan Inst Chem Eng. 2019;98:163-80.

[51] Bhuyan MS, Uddin MN, Islam MM, Bipasha FA, Hossain SS. Synthesis of graphene. Int Nano Lett. 2016;6(2):65-83.

[52] Jeon IY, Bae SY, Seo JM, Baek JB. Scalable production of edge-functionalized graphene nanoplatelets via mechanochemical ball-milling. Adv Funct Mater.

2015;25(45):6961-75.

[53] Eswaraiah V, Aravind SS, Ramaprabhu S. Top down method for synthesis of highly conducting graphene by exfoliation of graphite oxide using focused solar radiation. J Mater Chem. 2011;21(19):6800-3.

[54] Khan U, O'Neill A, Porwal H, May P, Nawaz K, Coleman JN. Size selection of dispersed, exfoliated graphene flakes by controlled centrifugation. Carbon. 2012;50(2):470-5.

[55] Gao E, Lin SZ, Qin Z, Buehler MJ, Feng XQ, Xu Z. Mechanical exfoliation of two-dimensional materials. J Mech Phys Solids. 2018;115:248-62.

[56] Lu X, Yu M, Huang H, Ruoff RS. Tailoring graphite with the goal of achieving single sheets. Nanotechnology. 1999;10(3):269.

[57] Lang BA. LEED study of the deposition of carbon on platinum crystal surfaces. Surf Sci. 1975;53(1):317-29.

[58] Wuest JD, Rochefort A. Strong adsorption of aminotriazines on graphene. Chem Commun. 2010;46(17):2923-5.

[59] Adetayo A, Runsewe D. Synthesis and fabrication of graphene and graphene oxide: A review. Open J Compos Mater. 2019;9(02):207.

[60] Zhou Q, Xia G, Du M, Lu Y, Xu H. Scotch-tape-like exfoliation effect of graphene quantum dots for efficient preparation of graphene nanosheets in water. Appl Surf Sci. 2019;483:52-9.

[61] Tung TT, Yoo J, Alotaibi FK, Nine MJ, Karunagaran R, Krebsz M, et al. Graphene oxide-assisted liquid phase exfoliation of graphite into graphene for highly conductive film and electromechanical sensors. ACS Appl Mater Inter. 2016;8(25):16521-32.

[62] Spasenovic M. Graphene market review. Graphene technology: From laboratory to fabrication. 1st ed. Hoboken, N): Wiley; 2016. p. 177-87

[63] Jayasena B, Subbiah S. A novel mechanical cleavage method for synthesizing few-layer graphenes. Nanoscale Res Lett. 2011;6(1):95.

[64] Chen J, Duan M, Chen G. Continuous mechanical exfoliation of graphene sheets via three-roll mill. J Mater Chem. 2012;22(37):19625-8.

[65] Liu L, Xiong Z, Hu D, Wu G, Chen P. Production of high quality single-or few-layered graphene by solid exfoliation of graphite in the presence of ammonia borane. Chem Commun. 2013;49(72):7890-2.

[66] Leon V, Quintana M, Herrero MA, Fierro JL, de la Hoz A, Prato $M$, et al. Few-layer graphenes from ball-milling of graphite with melamine. Chem Commun. 2011;47(39):10936-8.

[67] Yi M, Shen Z. A review on mechanical exfoliation for the scalable production of graphene. J Mater Chem A. 2015;3(22):11700-15.

[68] Shams SS, Zhang R, Zhu J. Graphene synthesis: A Review. Mater Sci Poland. 2015;33(3):566-78.

[69] Paton KR, Varrla E, Backes C, Smith RJ, Khan U, O'Neill A, et al. Scalable production of large quantities of defect-free few-layer graphene by shear exfoliation in liquids. Nat Mater. 2014;13(6):624-30.

[70] Rouzafzay F, Shidpour R, Al-Abri MZ, Qaderi F, Ahmadi A, Myint MT. Graphene@ ZnO nanocompound for short-time water treatment under sun-simulated irradiation: Effect of shear exfoliation of graphene using kitchen blender. J Alloy Compd. 2020;829:154614.

[71] Nepal A, Singh GP, Flanders BN, Sorensen CM. One-step synthesis of graphene via catalyst-free gas-phase hydrocarbon detonation. Nanotechnology. 2013;24(24):245602.

[72] Kairi MI, Dayou S, Kairi NI, Bakar SA, Vigolo B, Mohamed AR. Toward high production of graphene flakes: A review on recent developments in their synthesis methods and scalability. J Mater Chem A. 2018;6(31):15010-26.

[73] Viculis LM, Mack JJ, Mayer OM, Hahn HT, Kaner RB. Intercalation and exfoliation routes to graphite nanoplatelets. J Mater Chem. 2005;15(9):974-8.

[74] Vallés C, Drummond C, Saadaoui H, Furtado CA, He M, Roubeau 0 , et al. Solutions of negatively charged graphene sheets and ribbons. J Am Chem Soc. 2008;130(47):15802-4.

[75] Ciesielski A, Samori P. Graphene via sonication assisted liquid-phase exfoliation. Chem Soc Rev. 2014;43(1):381-98.

[76] Li Z, Young RJ, Backes C, Zhao W, Zhang X, Zhukov A, et al. Mechanisms of liquid-phase exfoliation for the production of graphene. ACS nano. 2020;14(9):10976-85.

[77] Cui X, Zhang C, Hao R, Hou Y. Liquid-phase exfoliation, functionalization and applications of graphene. Nanoscale. 2011;3(5):2118-26.

[78] Wang J, Zhao C, Mark LH, Wang X, Li R, Moghimian N, et al. Facilitating supercritical $\mathrm{CO}_{2}$ assisted exfoliation of graphene nanoplatelets with the polymer matrix. Chem Eng J. 2020;394:124930.

[79] Balaji SS, Karnan M, Kamarsamam J, Sathish M. Synthesis of boron-doped graphene by supercritical fluid processing and its application in symmetric supercapacitors using various electrolytes. Chem Electro Chem. 2019;6(5):1492-9.

[80] Hwang J, Park JH, Chung KY, Kim J. One-pot synthesis of Bireduced graphene oxide composite using supercritical acetone as anode for $\mathrm{Na}$-ion batteries. Chem Eng J. 2020;387:124111.

[81] Nursanto EB, Nugroho A, Hong SA, Kim SJ, Chung KY, Kim J. Facile synthesis of reduced graphene oxide in supercritical alcohols and its lithium storage capacity. Green Chem. 2011;13(10):2714-8.

[82] Yu P, Lowe SE, Simon GP, Zhong YL. Electrochemical exfoliation of graphite and production of functional graphene. Curr Opin Colloid Interface Sci. 2015;20(5-6):329-38. 
[83] Tseng KH, Ku HC, Tien DC, Stobinski L. Parameter control and concentration analysis of graphene colloids prepared by electric spark discharge method. Nanotechnol Rev. 2019;8(1):201-9.

[84] Zhong YL, Tian Z, Simon GP, Li D. Scalable production of graphene via wet chemistry: Progress and challenges. Mater Today. 2015;18(2):73-8.

[85] Mohan VB, Lau KT, Hui D, Bhattacharyya D. Graphene-based materials and their composites: A review on production, applications and product limitations. Compos Part B Eng. 2018;142:200-20.

[86] Chua CK, Pumera M. Chemical reduction of graphene oxide: A synthetic chemistry viewpoint. Chem Soc Rev. 2014;43(1):291-312.

[87] Kauppila J, Kunnas P, Damlin P, Viinikanoja A, Kvarnström C. Electrochemical reduction of graphene oxide films in aqueous and organic solutions. Electrochim Acta. 2013;89:84-9.

[88] Harima Y, Setodoi S, Imae I, Komaguchi K, Ooyama Y, Ohshita J, et al. Electrochemical reduction of graphene oxide in organic solvents. Electrochim Acta. 2011;56(15):5363-8.

[89] Chen L, Tang Y, Wang K, Liu C, Luo S. Direct electrodeposition of reduced graphene oxide on glassy carbon electrode and its electrochemical application. Electrochem Commun. 2011;13(2):133-7.

[90] Toh SY, Loh KS, Kamarudin SK, Daud WR. Graphene production via electrochemical reduction of graphene oxide: Synthesis and characterisation. Chem Eng J. 2014;251:422-34.

[91] Viinikanoja A, Wang Z, Kauppila J, Kvarnström C. Electrochemical reduction of graphene oxide and its in situ spectroelectrochemical characterization. Phys Chem Chem Phys. 2012;14(40):14003-9.

[92] Alanyalıoğlu M, Segura JJ, Oro-Sole J, Casan-Pastor N. The synthesis of graphene sheets with controlled thickness and order using surfactant-assisted electrochemical processes. Carbon. 2012;50(1):142-52.

[93] Toh SY, Loh KS, Kamarudin SK, Daud WR. Graphene production via electrochemical reduction of graphene oxide: Synthesis and characterisation. Chem Eng J. 2014;251:422-34.

[94] Guo HL, Wang XF, Qian QY, Wang FB, Xia XH. A green approach to the synthesis of graphene nanosheets. ACS Nano. 2009;3(9):2653-9.

[95] Cai M, Thorpe D, Adamson DH, Schniepp HC. Methods of graphite exfoliation. J Mater Chem. 2012; 22(48):24992-5002.

[96] Botas C, Álvarez P, Blanco C, Santamaría R, Granda M, Gutiérrez MD, et al. Critical temperatures in the synthesis of graphene-like materials by thermal exfoliation-reduction of graphite oxide. Carbon. 2013;52:476-85.

[97] Toh SY, Loh KS, Kamarudin SK, Daud WR. Graphene production via electrochemical reduction of graphene oxide: Synthesis and characterisation. Chem Eng J. 2014;251:422-34.

[98] Papageorgiou DG, Kinloch IA, Young RJ. Mechanical properties of graphene and graphene-based nanocomposites. Prog Mater Sci. 2017;90:75-127.

[99] Yan KA, Fu LE, Peng H, Liu Z. Designed CVD growth of graphene via process engineering. Acc Chem Res. 2013;46(10):2263-74.
[100] Yang Y, Han C, Jiang B, locozzia J, He C, Shi D, et al. Graphene-based materials with tailored nanostructures for energy conversion and storage. Mater Sci Eng R Rep. 2016;102:1-72.

[101] Jia K, Ci H, Zhang J, Sun Z, Ma Z, Zhu Y, et al. Superclean growth of graphene using a cold-wall chemical vapor deposition approach. Angew Chem Int Ed. 2020;59(39):17214-8.

[102] Boas CR, Focassio B, Marinho E, Larrude DG, Salvadori MC, Leão $C R$, et al. Characterization of nitrogen doped graphene bilayers synthesized by fast, low temperature microwave plasma-enhanced chemical vapour deposition. Sci Rep. 2019;9(1):1-2.

[103] Akimoto T, Ueno K. High crystallinity multilayer graphene deposited by a low-temperature CVD using $\mathrm{Ni}$ catalyst with applying current. 2019 Electron devices technology and manufacturing conference (EDTM). New York, US: IEEE; 2019 Mar 12. p. 351-53

[104] Sutter P. How silicon leaves the scene. Nat Mater. 2009;8(3):171-2.

[105] Norimatsu W, Kusunoki M. Epitaxial graphene on SiC \{0001\}: Advances and perspectives. Phys Chem Chem Phys. 2014;16(8):3501-11.

[106] Illakkiya JT, Rajalakshmi PU, Oommen R. Nebulized spray pyrolysis: A new method for synthesis of graphene film and their characteristics. Surf Coat Technol. 2016;307:65-72.

[107] Bianco A, Cheng HM, Enoki T, Gogotsi Y, Hurt RH, Koratkar N, et al. All in the graphene family-A recommended nomenclature for two-dimensional carbon materials. Carbon. 2013;65:1-6.

[108] Geim AK, Novoselov KS. The rise of graphene. Nanoscience and technology: A collection of reviews from nature journals. Berlin: Springer; 2010. p. 11-19

[109] Sinclair RC, Suter JL, Coveney PV. Micromechanical exfoliation of graphene on the atomistic scale. Phys Chem Chem Phys. 2019;21(10):5716-22.

[110] Parvez K, Yang S, Feng X, Müllen K. Exfoliation of graphene via wet chemical routes. Synth Met. 2015;210:123-32.

[111] Low CT, Walsh FC, Chakrabarti MH, Hashim MA, Hussain MA. Electrochemical approaches to the production of graphene flakes and their potential applications. Carbon. 2013; 54:1-21.

[112] Skaltsas T, Ke X, Bittencourt C, Tagmatarchis N. Ultrasonication induces oxygenated species and defects onto exfoliated graphene. J Phys Chem C. 2013;117(44):23272-8.

[113] Ober JA. Mineral commodity summaries 2016. US Geol Surv. 2016;1-202.

[114] Burchell TD, Pavlov TR. Graphite: Properties and characteristics. In: Konings RJ, Stoller RE, editors. Comprehensive nuclear materials. 2nd ed. Oxford: Elsevier; 2020. p. $355-81$

[115] Roy I, Sarkar G, Mondal S, Rana D, Bhattacharyya A, Saha NR, et al. Synthesis and characterization of graphene from waste dry cell battery for electronic applications. RSC Adv. 2016;6(13):10557-64.

[116] Tiwari SK, Huczko A, Oraon R, De Adhikari A, Nayak GC. Facile electrochemical synthesis of few layered graphene from discharged battery electrode and its application for energy storage. Arab J Chem. 2017;10(4):556-65. 
[117] Bandi S, Ravuri S, Peshwe DR, Srivastav AK. Graphene from discharged dry cell battery electrodes. J Hazard Mater. 2019;366:358-69.

[118] Adetayo A, Runsewe D. Synthesis and fabrication of graphene and graphene oxide: A review. Open J Compos Mater. 2019;9(02):207.

[119] Naghib SM, Behzad F, Rahmanian M, Zare Y, Rhee KY. A highly sensitive biosensor based on methacrylated graphene oxide-grafted polyaniline for ascorbic acid determination. Nanotechnol Rev. 2020;9(1):760-7.

[120] Seger B, Kamat PV. Electrocatalytically active grapheneplatinum nanocomposites. Role of 2-D carbon support in PEM fuel cells. J Phys Chem C. 2009;113(19):7990-5.

[121] Dideikin AT, Vul AY. Graphene oxide and derivatives: The place in graphene family. Front Phys. 2019;6:149.

[122] Seo DH, Yick S, Pineda S, Su D, Wang G, Han Z), et al. Singlestep, plasma-enabled reforming of natural precursors into vertical graphene electrodes with high areal capacitance. ACS Sustain Chem Eng. 2015;3(3):544-51.

[123] Seo DH, Yick S, Pineda S, Su D, Wang G, Han Z), et al. Singlestep, plasma-enabled reforming of natural precursors into vertical graphene electrodes with high areal capacitance. ACS Sustain Chem Eng. 2015;3(3):544-51.

[124] Seo DH, Rider AE, Han ZJ, Kumar S, Ostrikov K. Plasma break-down and Re-build: Same functional vertical graphenes from diverse natural precursors. Adv Mater. 2013;25(39):5638-42.

[125] Wu A, Li X, Yang J, Du C, Shen W, Yan J. Upcycling waste lard oil into vertical graphene sheets by inductively coupled plasma assisted chemical vapor deposition. Nanomaterials. 2017;7(10):318.

[126] Filip 0, Janda K, Kristoufek L, Zilberman D. Food versus fuel: An updated and expanded evidence. Energy Econ. 2019;82:152-66.

[127] Ravani F, Papagelis K, Dracopoulos V, Parthenios J, Dassios KG, Siokou A, et al. Graphene production by dissociation of camphor molecules on nickel substrate. Thin Solid Films. 2013;527:31-7.

[128] Kavitha K, Urade AR, Kaur G, Lahiri I. Low-temperature chemical vapor deposition growth of graphene layers on copper substrate using camphor precursor. J Nanosci Nanotechnol. 2020;20(12):7698-704.

[129] Chaliyawala HA, Rajaram N, Patel R, Ray A, Mukhopadhyay I. Controlled island formation of large-area graphene sheets by atmospheric chemical vapor deposition: Role of natural camphor. ACS Omega. 2019;4(5):8758-66.

[130] Kraus J, Böbel L, Zwaschka G, Günther S. Understanding the reaction kinetics to optimize graphene growth on $\mathrm{Cu}$ by chemical vapor deposition. Annalen der Phys. 2017;529(11):1700029.

[131] Somani PR, Somani SP, Umeno M. Planer nano-graphenes from camphor by CVD. Chem Phys Lett. 2006;430(1-3):56-9.

[132] Kalita G, Masahiro M, Uchida H, Wakita K, Umeno M. Few layers of graphene as transparent electrode from botanical derivative camphor. Mater Lett. 2010;64(20):2180-3.

[133] Kalita G, Wakita K, Umeno M. Monolayer graphene from a green solid precursor. Phys E Low Dimens Syst Nanostruct. 2011;43(8):1490-3.

[134] Jacob MV, Rawat RS, Ouyang B, Bazaka K, Kumar DS, Taguchi D, et al. Catalyst-free plasma enhanced growth of graphene from sustainable sources. Nano Lett.

2015;15(9):5702-8.

[135] Mohan A, Manoj B. Extraction of graphene nanostructures from Colocasia esculenta and Nelumbo nucifera leaves and surface functionalization with tin oxide: Evaluation of their antibacterial properties. Chem A Eur J. 2020;26(36):8105-14.

[136] Liu J, Rojas-Andrade MD, Chata G, Peng Y, Roseman G, Lu JE, et al. Photo-enhanced antibacterial activity of $\mathrm{ZnO} /$ graphene quantum dot nanocomposites. Nanoscale. 2018;10(1):158-66.

[137] Qu J, Luo C, Zhang Q, Cong Q, Yuan X. Easy synthesis of graphene sheets from alfalfa plants by treatment of nitric acid. Mater Sci Eng B. 2013;178(6):380-2.

[138] Roy P, Periasamy AP, Chuang C, Liou YR, Chen YF, Joly J, et al. Plant leaf-derived graphene quantum dots and applications for white LEDs. N J Chem. 2014;38(10):4946-51.

[139] Anooj ES, Praseetha PK. Synthesis and characterization of graphene quantum dots from nutmeg seeds and its biomedical application. Int J Recent Technol Eng. 2019;7:144-51.

[140] Kumar R, Singh RK, Singh DP. Natural and waste hydrocarbon precursors for the synthesis of carbon based nanomaterials: Graphene and CNTs. Renew Sustain Energy Rev. 2016;58:976-1006.

[141] Kumar R, Singh RK, Kumar P, Dubey PK, Tiwari RS, Srivastava ON. Clean and efficient synthesis of graphene nanosheets and rectangular aligned-carbon nanotubes bundles using green botanical hydrocarbon precursor: Sesame oil. Sci Adv Mater. 2014;6(1):76-83.

[142] Heli H, Yadegari H, Jabbari A. Graphene nanosheets-poly (o-aminophenol) nanocomposite for supercapacitor applications. Mater Chem Phys. 2012;134(1):21-5.

[143] Maarof S, Ali AA, Hashim AM. Synthesis of large-area singlelayer graphene using refined cooking palm oil on copper substrate by spray injector-assisted CVD. Nanoscale Res Lett. 2019;14(1):143.

[144] Abd Rahman SF, Mahmood MR, Hashim AM. Growth of uniform carbon thin film containing nanocrystalline graphene clusters from evaporated palm oil by thermal chemical vapor deposition. Japanese J Appl Phys. 2014;53(7):075101.

[145] Robaiah M, Mahmud MA, Salifairus MJ, Khusaimi Z, Azhan H, Abdullah S, et al. Synthesis and characterization of graphene from waste cooking palm oil at different deposition temperatures. AIP Conf Proc. 2019;2151(1):020026.

[146] Robaiah M, Rusop M, Abdullah S, Khusaimi Z, Azhan H, Laila MO, et al. Morphology and topography study of graphene synthesized from plant oil. AIP Conf Proc. 2018;1963(1):020045.

[147] Nasir S, Hussein MZ, Yusof NA, Zainal Z. Oil palm wastebased precursors as a renewable and economical carbon sources for the preparation of reduced graphene oxide from graphene oxide. Nanomaterials. 2017;7(7):182.

[148] Zhang B, Song J, Yang G, Han B. Large-scale production of high-quality graphene using glucose and ferric chloride. Chem Sci. 2014;5(12):4656-60.

[149] Wang X, Zhang Y, Zhi C, Wang X, Tang D, Xu Y, et al. Threedimensional strutted graphene grown by substrate-free sugar blowing for high-power-density supercapacitors. Nat Commun. 2013;4(1):1-8.

[150] Xu Z, Li Z, Holt CM, Tan X, Wang H, Amirkhiz BS, et al. Electrochemical supercapacitor electrodes from sponge-like 
graphene nanoarchitectures with ultrahigh power density. J Phys Chem Lett. 2012;3(20):2928-33.

[151] Xu Y, Lin Z, Huang X, Liu Y, Huang Y, Duan X. Flexible solidstate supercapacitors based on three-dimensional graphene hydrogel films. ACS Nano. 2013;7(5):4042-9.

[152] Park S, Mohanty N, Suk JW, Nagaraja A, An J, Piner RD, et al. Biocompatible, robust free-standing paper composed of a TWEEN/graphene composite. Adv Mater. 2010;22(15):1736-40.

[153] Akhavan O, Ghaderi E, Esfandiar A. Wrapping bacteria by graphene nanosheets for isolation from environment, reactivation by sonication, and inactivation by near-infrared irradiation. J Phys Chem B. 2011;115(19):6279-88.

[154] Akhavan O, Ghaderi E, Aghayee S, Fereydooni Y, Talebi A. The use of a glucose-reduced graphene oxide suspension for photothermal cancer therapy. J Mater Chem. 2012;22(27):13773-81.

[155] Wang Z, Ogata H, Morimoto S, Ortiz-Medina J, Fujishige M, Takeuchi K, et al. Nanocarbons from rice husk by microwave plasma irradiation: From graphene and carbon nanotubes to graphenated carbon nanotube hybrids. Carbon. 2015;94:479-84.

[156] Somanathan T, Prasad K, Ostrikov KK, Saravanan A, Krishna VM. Graphene oxide synthesis from agro waste. Nanomaterials. 2015;5(2):826-34.

[157] Shams SS, Zhang LS, Hu R, Zhang R, Zhu J. Synthesis of graphene from biomass: A green chemistry approach. Mater Lett. 2015;161:476-9.

[158] Adolfsson KH, Hassanzadeh S, Hakkarainen M. Valorization of cellulose and waste paper to graphene oxide quantum dots. RSC Adv. 2015;5(34):26550-8.

[159] Zhao H, Zhao TS. Graphene sheets fabricated from disposable paper cups as a catalyst support material for fuel cells. J Mater Chem A. 2013;1(2):183-7.

[160] Ruan G, Sun Z, Peng Z, Tour JM. Growth of graphene from food, insects, and waste. ACS Nano. 2011;5(9):7601-7.

[161] Titirici MM, White RJ, Brun N, Budarin VL, Su DS, del Monte F, et al. Sustainable carbon materials. Chem Soc Rev. 2015;44(1):250-90.

[162] Deng J, You Y, Sahajwalla V, Joshi RK. Transforming waste into carbon-based nanomaterials. Carbon. 2016;96:105-15.

[163] Lester E, Avila C, Pang CH, Williams O, Perkins J, Gaddipatti S, et al. A proposed biomass char classification system. Fuel. 2018;232:845-54.

[164] Yan Y, Meng Y, Tang L, Kostas ET, Lester E, Wu T, et al. Ignition and kinetic studies: The influence of lignin on biomass combustion. Energy Fuels. 2019;33(7):6463-72.

[165] Pang CH, Lester E, Wu T. Influence of lignocellulose and plant cell walls on biomass char morphology and combustion reactivity. Biomass Bioenerg. 2018;119:480-91.

[166] Myung Y, Jung S, Tung TT, Tripathi KM, Kim T. Graphenebased aerogels derived from biomass for energy storage and environmental remediation. ACS Sustain Chem Eng. 2019;7(4):3772-82.

[167] Bakar RA, Yahya R, Gan SN. Production of high purity amorphous silica from rice husk. Procedia Chem. 2016;19:189-95.

[168] Raghavan N, Thangavel S, Venugopal G. A short review on preparation of graphene from waste and bioprecursors. Appl Mater Today. 2017;7:246-54.
[169] Singh DP, Herrera CE, Singh B, Singh S, Singh RK, Kumar R. Graphene oxide: An efficient material and recent approach for biotechnological and biomedical applications. Mater Sci Eng C. 2018;86:173-97.

[170] Chen W, Lv G, Hu W, Li D, Chen S, Dai Z. Synthesis and applications of graphene quantum dots: A review. Nanotechnol Rev. 2018;7(2):157-85.

[171] Sun Z, Yan Z, Yao J, Beitler E, Zhu Y, Tour JM. Growth of graphene from solid carbon sources. Nature. 2010;468(7323):549-52.

[172] Takami T, Seino R, Yamazaki K, Ogino T. Graphene film formation on insulating substrates using polymer films as carbon source. J Phys D Appl Phys. 2014;47(9):094015.

[173] Kumar R, Singh RK, Singh DP, Joanni E, Yadav RM, Moshkalev SA. Laser-assisted synthesis, reduction and micro-patterning of graphene: Recent progress and applications. Coord Chem Rev. 2017;342:34-79.

[174] Tiliakos A, Ceaus C, Iordache SM, Vasile E, Stamatin I. Morphic transitions of nanocarbons via laser pyrolysis of polyimide films. J Anal Appl Pyrolysis. 2016;121:275-86.

[175] Ahmadi S, Afzalzadeh R. Few-layer graphene growth from polystyrene as solid carbon source utilizing simple APCVD method. Phys E Low Dimens Syst Nanostruct. 2016;81:302-7.

[176] Sharma S, Kalita G, Hirano R, Shinde SM, Papon R, Ohtani H, et al. Synthesis of graphene crystals from solid waste plastic by chemical vapor deposition. Carbon. 2014;72:66-73.

[177] Lin J, Peng Z, Liu Y, Ruiz-Zepeda F, Ye R, Samuel EL, et al. Laser-induced porous graphene films from commercial polymers. Nat Commun. 2014;5(1):1-8.

[178] Wang JZ, Zheng ZH, Li HW, Huck WT, Sirringhaus H. Dewetting of conducting polymer inkjet droplets on patterned surfaces. Nat Mater. 2004;3(3):171-6.

[179] Byun SJ, Lim H, Shin GY, Han TH, Oh SH, Ahn JH, et al. Graphenes converted from polymers. J Phys Chem Lett. 2011;2(5):493-7.

[180] Stankovich S, Piner RD, Chen X, Wu N, Nguyen ST, Ruoff RS. Stable aqueous dispersions of graphitic nanoplatelets via the reduction of exfoliated graphite oxide in the presence of poly (sodium 4-styrenesulfonate). J Mater Chem. 2006;16(2):155-8.

[181] Li D, Müller MB, Gilje S, Kaner RB, Wallace GG. Processable aqueous dispersions of graphene nanosheets. Nat Nanotechnol. 2008;3(2):101-5.

[182] Kim T, Lee H, Kim J, Suh KS. Synthesis of phase transferable graphene sheets using ionic liquid polymers. Acs Nano. 2010;4(3):1612-8.

[183] Kaushik PD, Ivanov IG, Lin PC, Kaur G, Eriksson J, Lakshmi GB, et al. Surface functionalization of epitaxial graphene on $\mathrm{SiC}$ by ion irradiation for gas sensing application. Appl Surf Sci. 2017;403:707-16.

[184] Shi Y, Zakharov AA, Ivanov IG, Yazdi GR, Jokubavicius V, Syväjärvi $M$, et al. Elimination of step bunching in the growth of large-area monolayer and multilayer graphene on off-axis 3CSiC (111). Carbon. 2018;140:533-42.

[185] Emtsev KV, Bostwick A, Horn K, Jobst J, Kellogg GL, Ley L, et al. Towards wafer-size graphene layers by atmospheric pressure graphitization of silicon carbide. Nat Mater. 2009;8(3):203-7. 
[186] Zhang H, Ding F, Li H, Qu F, Meng H, Gu H. Controlled synthesis of monolayer graphene with a high quality by pyrolysis of silicon carbide. Mater Lett. 2019;244:171-4.

[187] Zhao H, Ezeh Cl, Yin S, Xie Z, Pang CH, Zheng C, et al. $\mathrm{MoO}_{3^{-}}$ adjusted $\delta-\mathrm{MnO}_{2}$ nanosheet for catalytic oxidation of $\mathrm{Hg}^{\mathrm{O}}$ to $\mathrm{Hg}^{2+}$. Appl Catal B Environ. 2020;263:117829.

[188] Chen P, Xu C, Yin H, Gao X, Qu L. Shock induced conversion of carbon dioxide to few layer graphene. Carbon. 2017;115:471-6.

[189] Baik S, Park JH, Lee JW. One-pot conversion of carbon dioxide to CNT-grafted graphene bifunctional for sulfur cathode and thin interlayer of Li-S battery. Electrochim Acta. 2020;330:135264.

[190] Poh HL, Sofer Z, Luxa J, Pumera M. Transition metal-depleted graphenes for electrochemical applications via reduction of $\mathrm{CO}_{2}$ by lithium. Small. 2014;10(8):1529-35.

[191] Ambrosi A, Chua CK, Khezri B, Sofer Z, Webster RD, Pumera $M$. Chemically reduced graphene contains inherent metallic impurities present in parent natural and synthetic graphite. Proc Natl Acad Sci. 2012;109(32):12899-904.

[192] Guermoune A, Chari T, Popescu F, Sabri SS, Guillemette J, Skulason HS, et al. Chemical vapor deposition synthesis of graphene on copper with methanol, ethanol, and propanol precursors. Carbon. 2011;49(13):4204-10.

[193] Chen X, Xiang R, Zhao P, An H, Inoue T, Chiashi S, et al. Chemical vapor deposition growth of large single-crystal bernal-stacked bilayer graphene from ethanol. Carbon. 2016;107:852-6.

[194] Lisi N, Buonocore F, Dikonimos T, Leoni E, Faggio G, Messina G, et al. Rapid and highly efficient growth of graphene on copper by chemical vapor deposition of ethanol. Thin Solid Films. 2014;571:139-44.

[195] Campos-Delgado J, Botello-Méndez AR, Algara-Siller G, Hackens B, Pardoen T, Kaiser U, et al. CVD synthesis of mono-and few-layer graphene using alcohols at low hydrogen concentration and atmospheric pressure. Chem Phys Lett. 2013;584:142-6.

[196] Guermoune A, Chari T, Popescu F, Sabri SS, Guillemette J, Skulason HS, et al. Chemical vapor deposition synthesis of graphene on copper with methanol, ethanol, and propanol precursors. Carbon. 2011;49(13):4204-10.

[197] Faggio G, Capasso A, Messina G, Santangelo S, Dikonimos T, Gagliardi S, et al. High-temperature growth of graphene films on copper foils by ethanol chemical vapor deposition. J Phys Chem C. 2013;117(41):21569-76.

[198] Faggio G, Messina G, Lofaro C, Lisi N, Capasso A. Recent advancements on the CVD of graphene on copper from ethanol vapor C. J Carbon Res. 2020;6(1):14.

[199] Chen X, Zhao P, Xiang R, Kim S, Cha J, Chiashi S, et al. Chemical vapor deposition growth of $5 \mathrm{~mm}$ hexagonal singlecrystal graphene from ethanol. Carbon. 2015;94:810-5.

[200] Vijapur SH, Wang D, Botte GG. The growth of transparent amorphous carbon thin films from coal. Carbon. 2013;54:22-8.

[201] Daley PJ, Williams O, Heng Pang C, Wu T, Lester E. The impact of ash pellet characteristics and pellet processing parameters on ash fusion behaviour. Fuel. 2019;251:779-88.
[202] Trencher G, Healy N, Hasegawa K, Asuka J. Discursive resistance to phasing out coal-fired electricity: Narratives in Japan's coal regime. Energy Policy. 2019;132:782-96.

[203] Zhao H, Yang G, Pang CH, Fan H, Hall P, Kingman S, et al. Screening of metal oxidesfor $\mathrm{Hg}^{0}$ capture. Energy Procedia. 2015;75:2421-6.

[204] Vijapur SH, Wang D, Ingram DC, Botte GG. An investigation of growth mechanism of coal derived graphene films. Mater Today Commun. 2017;11:147-55.

[205] Powell C, Beall GW. Graphene oxide and graphene from low grade coal: Synthesis, characterization and applications. Curr Opin Colloid Interface Sci. 2015;20(5-6):362-6.

[206] Wang D, Vijapur SH, Botte GG. Coal char derived few-layer graphene anodes for lithium ion batteries. Photonics. 2014;1(3):251-9.

[207] Liu S, Xue J, Liu X, Chen H, Li X. Pitch derived graphene oxides: Characterization and effect on pyrolysis and carbonization of coal tar pitch. J Anal Appl Pyrolysis. 2020;145:104746.

[208] Pei S, Wei Q, Huang K, Cheng HM, Ren W. Green synthesis of graphene oxide by seconds timescale water electrolytic oxidation. Nat Commun. 2018;9(1):1-9.

[209] Liu W, Li H, Xu C, Khatami Y, Banerjee K. Synthesis of high-quality monolayer and bilayer graphene on copper using chemical vapor deposition. Carbon. 2011;49(13):4122-30.

[210] Chen Z, Ren W, Liu B, Gao L, Pei S, Wu ZS, et al. Bulk growth of mono-to few-layer graphene on nickel particles by chemical vapor deposition from methane. Carbon. 2010;48(12):3543-50.

[211] Lee I, Bae DJ, Lee WK, Yang CM, Cho SW, Nam J, et al. Rapid synthesis of graphene by chemical vapor deposition using liquefied petroleum gas as precursor. Carbon. 2019;145:462-9.

[212] Liu WW, Chai SP, Mohamed AR, Hashim U. Synthesis and characterization of graphene and carbon nanotubes: $A$ review on the past and recent developments. J Ind Eng Chem. 2014;20(4):1171-85.

[213] Omrani AN, Esmaeilzadeh E, Jafari M, Behzadmehr A. Effects of multi walled carbon nanotubes shape and size on thermal conductivity and viscosity of nanofluids. Diam Relat Mater. 2019;93:96-104.

[214] Muller J, Delos M, Panin N, Rabolli V, Huaux F, Lison D. Absence of carcinogenic response to multiwall carbon nanotubes in a 2-year bioassay in the peritoneal cavity of the rat. Toxicol Sci. 2009;110(2):442-8.

[215] Silva AA, Pinheiro RA, Rodrigues AC, Baldan MR, TravaAiroldi VJ, Corat EJ. Graphene sheets produced by carbon nanotubes unzipping and their performance as supercapacitor. Appl Surf Sci. 2018;446:201-8.

[216] Tiwary CS, Javvaji B, Kumar C, Mahapatra DR, Ozden S, Ajayan PM, et al. Chemical-free graphene by unzipping carbon nanotubes using cryo-milling. Carbon. 2015;89:217-24.

[217] Kosynkin DV, Higginbotham AL, Sinitskii A, Lomeda JR, Dimiev A, Price BK, et al. Longitudinal unzipping of carbon 
nanotubes to form graphene nanoribbons. Nature. 2009;458(7240):872-6.

[218] Saghafi M, Mahboubi F, Mohajerzadeh S, Fathi M, Holze R. Longitudinal unzipping of carbon nanotubes and their electrochemical performance in supercapacitors. Curr Appl Phys. 2014;14(10):1335-43.

[219] Xiao B, Li X, Li X, Wang B, Langford C, Li R, et al. Graphene nanoribbons derived from the unzipping of carbon nanotubes: Controlled synthesis and superior lithium storage performance. J Phys Chem C. 2014;118(2):881-90.
[220] Liu Q, Shi Y, Zheng S, Ning L, Ye Q, Tao M, et al. Aminefunctionalized low-cost industrial grade multi-walled carbon nanotubes for the capture of carbon dioxide. J Energy Chem. 2014;23(1):111-8.

[221] Oliveira ML, Izquierdo M, Querol X, Lieberman RN, Saikia BK, Silva LF. Nanoparticles from construction wastes: A problem to health and the environment. J Clean Prod. 2019;219:236-43.

[222] Kim J, Lee G, Kim J. Wafer-scale synthesis of multi-layer graphene by high-temperature carbon ion implantation. Appl Phys Lett. 2015;107(3):033104. 